\title{
Some Inequalities for a New Class of Convex Functions with Applications via Local Fractional Integral
}

\author{
Hu Ge-JiLe, ${ }^{1}$ Saima Rashid $\mathbb{D},{ }^{2}$ Fozia Bashir Farooq, ${ }^{3}$ and Sobia Sultana ${ }^{3}$ \\ ${ }^{1}$ School of Science, Huzhou University, Huzhou 313000, China \\ ${ }^{2}$ Department of Mathematics, Government College University, Faisalabad, Pakistan \\ ${ }^{3}$ Imam Mohammad Ibn Saud Islamic University, Riyadh, KSA, Saudi Arabia \\ Correspondence should be addressed to Saima Rashid; saimarashid@gcuf.edu.pk
}

Received 10 December 2020; Revised 28 January 2021; Accepted 10 March 2021; Published 2 April 2021

Academic Editor: Shanhe Wu

Copyright (c) $2021 \mathrm{Hu}$ Ge-JiLe et al. This is an open access article distributed under the Creative Commons Attribution License, which permits unrestricted use, distribution, and reproduction in any medium, provided the original work is properly cited.

The understanding of inequalities in convexity is crucial for studying local fractional calculus efficiency in many applied sciences. In the present work, we propose a new class of harmonically convex functions, namely, generalized harmonically $\psi$-s-convex functions based on fractal set technique for establishing inequalities of Hermite-Hadamard type and certain related variants with respect to the Raina's function. With the aid of an auxiliary identity correlated with Raina's function, by generalized Hölder inequality and generalized power mean, generalized midpoint type, Ostrowski type, and trapezoid type inequalities via local fractional integral for generalized harmonically $\psi$-s-convex functions are apprehended. The proposed technique provides the results by giving some special values for the parameters or imposing restrictive assumptions and is completely feasible for recapturing the existing results in the relative literature. To determine the computational efficiency of offered scheme, some numerical applications are discussed. The results of the scheme show that the approach is straightforward to apply and computationally very user-friendly and accurate.

\section{Introduction}

Fractional calculus is a developing arena in mathematics with profound presentations in all connected areas such as wave liquids, thermodynamics, image processing, virology, biological population models, chaos, and signals processing, see [15]. It has been employed to model physical phenomena that are originated to be paramountly described by fractional differential equations. Nowadays, the local fractional calculus is trying to report the nondifferentiable problems, for example, heat conduction problem involving local derivatives of fractional order [6,7], local fractional Tricomi equation [8], and fractal vehicular traffic flow [9].

Also, several noted variants via local fractional integral have been investigated by many researchers, for example, Mo et al. [10] propounded Hermite-Hadamard inequalities for generalized $s$-convex functions. Abdeljawad et al. [11] introduced some local fractional variants for generalized $(s$, $m$ )-convex functions in the fractal style with applications.
Also, Sun and Liu [12] reported new generalizations of Hermite-Hadamard inequalities for generalized harmonically convex functions. For further investigations concerning to local fractional integral inequalities, we recommend the readers to [13-15] and the references therein.

It is well known that convexity theory has potential applications in many intriguing and captivating fields of research and furthermore played a remarkable role in numerous areas, such as coding theory, optimization, physics, information theory, engineering, and inequality theory. Several new classes of classical convexity have been proposed in the literature (see [16-26]). Many researchers endeavored, attempted, and maintain their work on the concept of convex functions, generalize its variant forms in different ways using innovative ideas and fruitful techniques [27-29]. In this regard, integral inequalities have played an important role in describing real world problems such as in elementary research conducted by Niculescu and Persson [30]. In this context, HermiteHadamard $[31,32]$ like type inequalities are very influential 
in convexity theory, which describes that if $Q: \rrbracket \mapsto \mathbb{R}$ is convex function, then the double inequality

$$
Q\left(\frac{a_{1}+a_{2}}{2}\right) \leq \frac{1}{a_{2}-a_{1}} \int_{a_{1}}^{a_{2}} Q(z) d z \leq \frac{Q\left(a_{1}\right)+Q\left(a_{2}\right)}{2},
$$

holds for all $a_{1}, a_{2} \in \llbracket$ with $a_{1} \neq a_{2}$.

Numerous approaches have been done by several researchers to inquire novel generalizations, speculations, and modifications for the Hermite-Hadamard inequality and its variant forms; we prescribe the related phenomena [33-36] to interested readers.

Now, we evoke the concept of harmonically convex function [37] as follows.

Definition 1 ([37]). Let $\mathbb{H} \subset \mathbb{R}$ be an interval, and a realvalued function $\mathrm{Q}: \mathbb{H} \mapsto \mathbb{R}$ is said to be harmonically convex if the inequality

$$
Q\left(\frac{a_{1} a_{2}}{t_{1} a_{1}+\left(1-t_{1}\right) a_{2}}\right) \leq\left(1-t_{1}\right) Q\left(a_{1}\right)+t_{1} Q\left(a_{2}\right)
$$

holds for all $a_{1}, a_{2} \in \mathbb{H}$ and $t_{1} \in[0,1]$.

In [37], İscan derived the Hermite-Hadamard inequality for harmonically convex function.

$$
Q\left(\frac{2 a_{1} a_{2}}{a_{1}+a_{2}}\right) \leq \frac{a_{1} a_{2}}{a_{2}-a_{1}} \int_{a_{1}}^{a_{2}} \frac{Q(z)}{z^{2}} d z \leq \frac{Q\left(a_{1}\right)+Q\left(a_{2}\right)}{2},
$$

holds for all $a_{1}, a_{2} \in \mathbb{H}$ with $a_{1}<a_{2}$ and $Q \in L_{1}\left[a_{1}, a_{2}\right]$ (the set of Lebesgue integrable functions).

To make this collection more comprehensive, we introduce the notion of generalized harmonically $\psi$-s-convex functions in the sense of Raina's function on fractal set. By considering generalized harmonically $\psi$-s-convex functions, certain refinements of Hermite-Hadamard type inequalities are derived that can be used to characterize some useful consequences of harmonically $\psi$-convex functions with the appropriate assumption of $\widehat{\alpha}$, which are connected to Raina's function. Remarkable special cases are captured, and new Ostrowski type results are proved for the aforesaid class of functions. Additionally, our findings for the new schemes are also constituted some better approaches in various directions for proposing variation in $\widehat{\alpha}$, accordingly Remark 12 . Application of the $\widehat{\alpha}$-type special mean and Mittag-Leffler functions are presented by mingling the suggested methodology and the theory of the special functions. We hope that the idea and results obtained herein will be a catalyst for further investigation.

\section{Prelude}

In what follows, we demonstrate a different notion of differentiation that merges the idea of fractional differentiation and fractal derivative; recently, one has established the accompanying sets [6].
For the sake of brevity, we mention $\mathbb{N}$ (the set of natural numbers), $\mathbb{Z}$ (set of positive integers), $\mathbb{Q}$ (set of rational number), and $\mathbb{R}$ (set of real numbers), respectively.

Also, whenever the $\widehat{\alpha}$-type set $\mathbb{R}^{\widehat{\alpha}}$ of real line numbers are included, the $\widehat{\alpha}$ is supposed to be implicitly $0<\widehat{\alpha} \leq 1$. One has also characterized by two binary operations the addition "+" and the multiplication "." (which is conventionally omitted) on the $\widehat{\alpha}$-type set $\mathbb{R}^{\widehat{\alpha}}$ of real line numbers as follows.

For $e_{1}^{\widehat{\alpha}}, \widehat{e_{2}^{\alpha}} \in \mathbb{R}^{\widehat{\alpha}}$, two binary operations the addition “+” and the multiplication "." are defined as $e_{1}^{\widehat{\alpha}}+e_{2}^{\widehat{\alpha}}:=\left(e_{1}+e_{2}\right)^{\widehat{\alpha}}$ and $e_{1}^{\widehat{\alpha}} \cdot e_{2}^{\widehat{\alpha}}:=\left(e_{1} \cdot e_{2}\right)^{\widehat{\alpha}}$, for $e_{1}^{\widehat{\alpha}}, e_{2}^{\widehat{\alpha}} \in \mathbb{R}^{\widehat{\alpha}}$.

Then, we have the following asserations to show

(1) $\left(\mathbb{R}^{\widehat{\alpha}},+\right)$ is an abelian group: for $e_{1}^{\widehat{\alpha}}, e_{2}^{\widehat{\alpha}}, e_{3}^{\widehat{\alpha}} \in \mathbb{R}^{\widehat{\alpha}}$

(i) $e_{1}^{\widehat{\alpha}}+e_{2}^{\widehat{\alpha}} \in \mathbb{R}^{\widehat{\alpha}}$

(ii) $e_{1}^{\widehat{\alpha}}+e_{2}^{\widehat{\alpha}}=e_{2}^{\widehat{\alpha}}+e_{1}^{\widehat{\alpha}}$

(iii) $e_{1}^{\widehat{\alpha}}+\left(e_{2}^{\widehat{\alpha}}+e_{3}^{\widehat{\alpha}}\right)=\left(e_{1}^{\alpha}+e_{2}^{\widehat{\alpha}}\right)+e_{3}^{\widehat{\alpha}}$

(iv) $0^{\widehat{\alpha}}+e_{1}^{\widehat{\alpha}}=e_{1}^{\widehat{\alpha}}+0^{\widehat{\alpha}}=e_{1}^{\widehat{\alpha}} ; 0^{\widehat{\alpha}}$ is the additive identity for $\left(\mathbb{R}^{\alpha},+\right)$

(v) $e_{1}^{\widehat{\alpha}}+\left(-e_{1}^{\widehat{\alpha}}\right)=\left(-e_{1}^{\widehat{\alpha}}\right)+e_{1}^{\widehat{\alpha}}=0^{\widehat{\alpha}}$; for any $e^{\widehat{\alpha}} \in \mathbb{R}^{\widehat{\alpha}}$, $-e^{\alpha}$ is the inverse element of $e^{\alpha}$ for $\left(\mathbb{R}^{\alpha},+\right)$

(2) $\left(\mathbb{R}^{\widehat{\alpha}} \backslash\left\{0^{\widehat{\alpha}}\right\}\right.$, .) is an abelian group: for $e_{1}^{\widehat{\alpha}}, e_{2}^{\widehat{\alpha}}, e_{3}^{\alpha} \in \mathbb{R}^{\widehat{\alpha}}$

(vi) $e_{1}^{\widehat{\alpha}} \cdot e_{2}^{\alpha} \in \mathbb{R}^{\widehat{\alpha}}$

(vii) $e_{1}^{\widehat{\alpha}} \cdot e_{2}^{\widehat{\alpha}}=e_{2}^{\widehat{\alpha}} \cdot \widehat{e} e_{1}^{\alpha}$

(viii) $e_{1}^{\widehat{\alpha}} \cdot\left(e_{2}^{\widehat{\alpha}} \cdot e_{3}^{\widehat{\alpha}}\right)=\left(e_{1}^{\widehat{\alpha}} \cdot e_{2}^{\widehat{\alpha}}\right) \cdot e_{3}^{\widehat{\alpha}}$

(ix) $1^{\widehat{\alpha}}+e_{1}^{\widehat{\alpha}}=e_{1}^{\widehat{\alpha}}+1^{\widehat{\alpha}}=e_{1}^{\widehat{\alpha}} ; 1^{\widehat{\alpha}}$ is the multiplicative identity for $\left(\mathbb{R}^{\widehat{\alpha}},.\right)$

(x) $e_{1}^{\widehat{\alpha}}\left(1 / e_{1}\right)^{\widehat{\alpha}}=\left(1 / e_{1}\right)^{\widehat{\alpha}} e_{1}^{\widehat{\alpha}}=1^{\widehat{\alpha}} ;$ for any $e^{\widehat{\alpha}} \in \mathbb{R}^{\widehat{\alpha}}$ $\backslash\left\{0^{\widehat{\alpha}}\right\},(1 / e)^{\widehat{\alpha}}$ is the inverse element of $e^{\widehat{\alpha}}$ for $\left(\mathbb{R}^{\widehat{\alpha}},.\right)$

(3) Distributive law holds: $e_{1}^{\widehat{\alpha}}\left(e_{2}^{\widehat{\alpha}}+e_{3}^{\widehat{\alpha}}\right)=e_{1}^{\widehat{\alpha}} e_{2}^{\widehat{\alpha}}+e_{1}^{\widehat{\alpha}} e_{3}^{\widehat{\alpha}}$

Proposition 2. The following asserations holds true:

(i) $\left(\mathbb{R}^{\widehat{\alpha}},+,.\right)$ is a field

(ii) The additive identity $0^{\widehat{\alpha}}$ and the multiplication identity $1^{\widehat{\alpha}}$ are unique

(iii) The additive inverse element and the multiplicative inverse element are unique 
(iv) For each $e_{1}^{\widehat{\alpha}} \in \mathbb{R}^{\widehat{\alpha}} \backslash\left\{0^{\widehat{\alpha}}\right\}$, its inverse element $\left(1 / e_{1}\right)^{\widehat{\alpha}}$ may be written as $1^{\alpha} / e_{1}^{\widehat{\alpha}}$ but not as $1 / e_{1}^{\widehat{\alpha}}$; for each $e_{1}^{\widehat{\alpha}} \in \mathbb{R}^{\widehat{\alpha}}$, its inverse element $\left(-e_{1}\right)^{\widehat{\alpha}}$ may be written as $-e_{1}^{\widehat{\alpha}}$

(v) If the order $<$ is defined on $\left(\mathbb{R}^{\widehat{\alpha}},+\right)$ as follows: $e_{1}^{\widehat{\alpha}}<$ $\widehat{e_{2}^{\alpha}} \in \mathbb{R}^{\widehat{\alpha}}$ if and only if $e_{1}<e_{2} \in \mathbb{R}$, then $\left(\mathbb{R}^{\widehat{\alpha}},+, .,<\right)$ is an ordered field like $(\mathbb{R},+, .,<)$

In [6], Yang introduced the following notions for local fractional continuity and differentiability as follows:

Definition 3 ([6]). A nondifferentiable mapping $Q: \mathbb{R} \rightarrow$ $\mathbb{R}^{\widehat{\alpha}}, t_{1} \rightarrow Q(\varepsilon)$ is said to be local fractional continuous at $\varepsilon_{\circ}$, if for any $\varepsilon>0$, there exists $m>0$, satisfying that

$$
\left|Q(\varepsilon)-Q\left(\varepsilon_{\circ}\right)\right|<\varepsilon^{\widehat{\alpha}},
$$

holds for $\left|\varepsilon-\varepsilon_{\circ}\right|<m$. If $Q(\varepsilon)$ is local continuous on $\left(a_{1}, a_{2}\right)$, then we symbolize it by $Q(\varepsilon) \in \mathbb{C}_{\widehat{\alpha}}\left(a_{1}, a_{2}\right)$.

Definition 4 ([6]). The local fractional derivative of $Q(\varepsilon)$ of order $\widehat{\alpha}$ at $\varepsilon=\varepsilon_{\mathrm{o}}$ is defined by the expression

$$
Q^{(\widehat{\alpha})}\left(\varepsilon_{\circ}\right)={ }_{\varepsilon_{\circ}} \mathscr{D}_{\varepsilon}^{\widehat{\alpha}} Q(\varepsilon)=\left.\frac{d^{\widehat{\alpha}} Q(\varepsilon)}{d \varepsilon^{\widehat{\alpha}}}\right|_{\varepsilon=\varepsilon_{\circ}}=\lim _{\varepsilon \rightarrow \varepsilon_{\circ}} \frac{\Delta^{\widehat{\alpha}}\left(Q(\varepsilon)-Q\left(\varepsilon_{\circ}\right)\right)}{\left(\varepsilon-\varepsilon_{\circ}\right)^{\widehat{\alpha}}},
$$

where $\Delta^{\widehat{\alpha}}\left(Q(\varepsilon)-Q\left(\varepsilon_{\circ}\right)\right)=\Gamma(\widehat{\alpha}+1)\left(Q(\varepsilon)-Q\left(\varepsilon_{\circ}\right)\right)$. Let $Q^{(\widehat{\alpha})}$ $(\varepsilon)=\mathscr{D}_{\varepsilon}^{\widehat{\alpha}} Q(\varepsilon)$. If there exists $Q^{(k+1) \widehat{\alpha}}(\varepsilon)=\overbrace{\mathscr{D}_{\varepsilon}^{\alpha} \ldots \mathscr{D}_{\varepsilon}^{\alpha}}^{(k+1) \text { times }} Q(\varepsilon)$ for any $\varepsilon \in \Omega \subseteq \mathbb{R}$, then it is denoted by $Q \in \mathscr{D}_{(k+1) \widehat{\alpha}}(\mathscr{I})$, where $k=0,1,2, \cdots$.

Definition 5. Let $Q(\varepsilon) \in \mathbb{C}_{\widehat{\alpha}}\left[a_{1}, a_{2}\right]$, and let $\Delta=\left\{\rho_{0}, \rho_{1}, \cdots\right.$, $\left.\rho_{N}\right\},(N \in \mathbb{N})$ be a partition of $\left[a_{1}, a_{2}\right]$ which satisfies $a_{1}=$ $\rho_{0}<\rho_{1}<\cdots<\rho_{N}=a_{2}$. Then, the local fractional integral of $Q$ on $\left[a_{1}, a_{2}\right]$ of order $\widehat{\alpha}$ is defined as follows

$$
\begin{aligned}
a_{a_{1}} \mathscr{I}_{a_{2}}^{(\widehat{\alpha})} Q(\varepsilon) & =\frac{1}{\Gamma(1+\widehat{\alpha})} \int_{a_{1}}^{a_{2}} Q(\rho)(d \rho)^{\widehat{\alpha}} \\
& :=\frac{1}{\Gamma(1+\widehat{\alpha})} \lim _{\delta \rho \rightarrow 0} \sum_{j=0}^{N-1} Q\left(\rho_{j}\right)\left(\Delta \rho_{j}\right),
\end{aligned}
$$

where $\delta \rho:=\max \left\{\Delta \rho_{1}, \Delta \rho_{2}, \cdots, \Delta \rho_{N-1}\right\}$ and $\Delta \rho_{j}:=\rho_{j+1}-\rho_{j}$, $j=0, \cdots, N-1$.

Here, it follows that ${ }_{a_{1}} \mathcal{F}_{a_{2}}^{(\widehat{\alpha})} Q(\varepsilon)=0$ if $a_{1}=a_{2}$ and $a_{1}$ $\mathscr{I}_{a_{2}}^{(\widehat{\alpha})} Q(\varepsilon)={ }_{a_{2}} \mathscr{I}_{a_{1}}^{(\widehat{\alpha})} Q(\varepsilon)$ if $a_{1}<a_{2}$. For any $\varepsilon \in\left[a_{1}, a_{2}\right]$, if there exists ${ }_{a_{1}} \mathscr{F}_{a_{2}}^{(\widehat{\alpha})} Q(\varepsilon)$, then it is denoted by $Q(\varepsilon) \epsilon$ $\mathcal{F}_{\varepsilon}^{\widehat{\alpha}}\left[a_{1}, a_{2}\right]$.

\section{Lemma 6 ([6]).}

(1) Suppose that $Q(z)=Q^{(\widehat{\alpha})}(z) \in \mathbb{C}_{\widehat{\alpha}}\left[a_{1}, a_{2}\right]$, then

$$
{ }_{a_{1}} \mathcal{I}_{a_{2}}^{(\widehat{\alpha})} Q(z)=Q\left(a_{2}\right)-Q\left(a_{1}\right) \text {. }
$$

(2) Suppose that $Q(z), W(z) \in \mathscr{D}_{\widehat{\alpha}}\left[a_{1}, a_{2}\right]$, and $Q^{(\widehat{\alpha})}(z)$, $W^{(\widehat{\alpha})}(z) \in \mathbb{C}_{\widehat{\alpha}}\left[a_{1}, a_{2}\right]$, then

$$
{ }_{a_{1}} \mathcal{I}_{a_{2}}^{(\widehat{\alpha})} \mathrm{Q}(z) W^{(\widehat{\alpha})}(z)=\left.Q(u) W(z)\right|_{a_{1}} ^{a_{2}}{ }_{a_{1}} \mathcal{I}_{a_{2}}^{(\widehat{\alpha})} Q^{(\widehat{\alpha})}(z) W(z)
$$

\section{Lemma 7 ([6]).}

$$
\begin{aligned}
\frac{d^{\widehat{\alpha}} z^{k \widehat{\alpha}}}{d z^{\widehat{\alpha}}} & =\frac{\Gamma(1+k \widehat{\alpha})}{\Gamma(1+(k-1) \widehat{\alpha})} z^{(k-1) \widehat{\alpha}}, \frac{1}{\Gamma(1+\widehat{\alpha})} \int_{a_{1}}^{a_{2}} z^{k \widehat{\alpha}}(d z)^{\widehat{\alpha}} \\
& =\frac{\Gamma(1+k \widehat{\alpha})}{\Gamma(1+(k+1) \widehat{\alpha})}\left(a_{2}^{(k+1) \widehat{\alpha}}-a_{1}^{(k+1) \widehat{\alpha}}\right), k>0 .
\end{aligned}
$$

An analog in the fractal set $\mathbb{R}^{\widehat{\alpha}}$ of the classical Hölder's inequality has been established by [15], which is asserted by the following lemma.

Lemma 8 ([15]. Generalized Hölder's inequality). For $\omega_{1}, \omega_{2}$ $>1$ with $\omega_{1}^{-1}+\omega_{2}^{-1}=1$, and let $Q, W \in \mathbb{C}_{\widehat{\alpha}}\left[a_{1}, a_{2}\right]$, then

$$
\begin{aligned}
\frac{1}{\Gamma(1+\widehat{\alpha})} \int_{a_{1}}^{a_{2}}|Q(z) W(z)|(d z)^{\widehat{\alpha}} \leq & \left(\frac{1}{\Gamma(1+\widehat{\alpha})} \int_{a_{1}}^{a_{2}}|Q(z)|^{\omega_{1}}(d z)^{\widehat{\alpha}}\right)^{1 / \omega_{1}} \\
& \cdot\left(\frac{1}{\Gamma(1+\widehat{\alpha})} \int_{a_{1}}^{a_{2}}|W(z)|^{\omega_{2}}(d z)^{\widehat{\alpha}}\right)^{1 / \omega_{2}} .
\end{aligned}
$$

In the work of Sun and Liu [13], we present the following concept related with the notion of generalized harmonically convexity.

Definition 9 ([13]). Let $\mathbb{H} \subset(0, \infty)$ be an interval and let then $Q: \mathbb{H} \mapsto \mathbb{R}^{\widehat{\alpha}}(0<\widehat{\alpha} \leq 1)$ is said to be generalized harmonically convex function if the inequality holds:

$$
Q\left(\frac{a_{1} a_{2}}{t_{1} a_{1}+\left(1-t_{1}\right) a_{2}}\right) \leq \widehat{t_{1}^{\widehat{\alpha}}} Q\left(a_{1}\right)+\left(1-t_{1}\right)^{\widehat{\alpha}} Q\left(a_{2}\right),\left(\forall a_{1}, a_{2} \in \mathbb{H}, t_{1} \in[0,1]\right) \text {. }
$$

Recently, several noted consequences and motivating facts related to fractal theory have been appeared in the 
literature $[5,14,15]$. In [13], Sun explored the following ana$\log$ of the Hermite-Hadamard inequality (3) for generalized harmonically convex functions.

$$
\begin{aligned}
\frac{1}{\Gamma(1+\widehat{\alpha})} Q\left(\frac{2 a_{1} a_{2}}{a_{1}+a_{2}}\right) & \leq\left(\frac{a_{1} a_{2}}{a_{2}-a_{1}}\right)_{a_{1}}^{\widehat{\alpha}} \mathscr{I}_{a_{2}}^{(\widehat{\alpha})} \frac{Q(z)}{z^{2 \widehat{\alpha}}} \\
& \leq \frac{\Gamma(1+\widehat{\alpha})}{\Gamma(1+2 \widehat{\alpha})}\left[Q\left(a_{1}\right)+Q\left(a_{2}\right)\right] .
\end{aligned}
$$

Firstly, let $\lambda=\{\lambda(m)\}_{m=0}^{\infty}$ be a bounded sequence of real numbers, and $\mathscr{F}_{\rho, \sigma}^{\lambda}(.) \rho, \sigma>0$ denotes the Raina's function.

In [38], Raina explored a new class of functions stated as:

$$
\mathscr{F}_{\rho, \sigma}^{\lambda}(z)=\mathscr{F}_{\rho, \sigma}^{\lambda(0), \lambda(1), \cdots}(z)=\sum_{m=0}^{\infty} \frac{\lambda(m)}{\Gamma(\rho m+\lambda)} z^{m}
$$

where $\rho, \sigma>0,|z|<\mathbb{R}$ and

$$
\lambda=(\lambda(0), \cdots, \lambda(m), \cdots)
$$

is bounded sequence of positive real numbers. Notice that if we choose $\rho=1, \sigma=0$ in (13), then

$$
\lambda(m)=\frac{\left(\delta_{1}\right)_{m}\left(\delta_{2}\right)_{m}}{\left(\delta_{3}\right)_{m}} \text { for } m=0,1,2, \cdots
$$

where $\delta_{1}, \delta_{2}$, and $\delta_{3}$ are parameters which can choose aribtrary real and complex values (provided that $\delta_{3} \neq 0,-1,-2$, $\cdots$, ), and we have the notion $(b)_{m}$ by

$$
(b)_{m}=\frac{\Gamma(b+m)}{\Gamma(b)}=b(b+1) \cdots(b+m-1), m=0,1,2, \cdots
$$

then the classical hypergeometric function stated as follows

$$
\mathscr{F}_{\rho, \sigma}^{\lambda}(z)=F\left(\delta_{1}, \delta_{2} ; \delta_{3} ; z\right)=\sum_{m=0}^{\infty} \frac{\left(\delta_{1}\right)_{m}\left(\delta_{2}\right)_{m}}{m !\left(\delta_{3}\right)_{m}} z^{m},|z| \leq 1, z \in \mathbb{C} .
$$

Also, if $\lambda=(1,1, \cdots)$ with $\varsigma=\delta,(\Re(\delta)>0), \lambda=1$ and restricting its domain to $z \in \mathbb{C}$ in (13), then we have the classical Mittag-Leffler function:

$$
E_{\delta_{1}}(z)=\sum_{m=0}^{\infty} \frac{1}{\Gamma\left(1+\delta_{1} m\right)} z^{m}
$$

Next, we evoke a novel concept of harmonic set and mappings including Raina's functions.
Definition 10. Let $\rho, \sigma>0$ and $\lambda=\{\lambda(m)\}_{m=0}^{\infty}$ be a bounded sequence of real numbers. A nonempty set $\mathbb{H}_{\mathscr{F}} \subset(0, \infty)$ is said to be generalized harmonically $\psi$-convex set, if

$$
\frac{a_{1}\left(a_{1}+\mathscr{F}_{\rho, \sigma}^{\lambda}\left(a_{2}-a_{1}\right)\right)}{a_{1}+t_{1} \mathscr{F}_{\rho, \sigma}^{\lambda}\left(a_{2}-a_{1}\right)} \in \mathbb{H}_{\mathscr{F}}
$$

for all $a_{1}, a_{2} \in \mathbb{H}_{\mathscr{F}}, t_{1} \in[0,1]$.

Next, we introduce the notion of generalized harmonically $\psi$-s-convex function as follows:

Definition 11. Let $\rho, \sigma>0$ and $\lambda=\{\lambda(m)\}_{m=0}^{\infty}$ be a bounded sequence of real numbers. If a mapping $Q: \mathbb{H}_{\mathscr{F}} \mapsto \mathbb{R}^{\widehat{\alpha}}(0 \leq$ $\widehat{\alpha}<1)$ satisfies the following inequality:

$Q\left(\frac{a_{1}\left(a_{1}+\mathscr{F}_{\rho, \sigma}^{\lambda}\left(a_{2}-a_{1}\right)\right)}{a_{1}+t_{1} \mathscr{F}_{\rho, \sigma}^{\lambda}\left(a_{2}-a_{1}\right)}\right) \leq t_{1}^{\widehat{s \alpha}} Q\left(a_{1}\right)+\left(1-t_{1}\right)^{\widehat{s \alpha}} Q\left(a_{2}\right)$,

holds for all $a_{1}, a_{2} \in \mathbb{H}_{\mathscr{F}}, t_{1} \in[0,1]$ and $s \in(0,1]$.

Remark 12. In view of Definition 11, we have that

(i) Choosing $\widehat{\alpha}=1$, then we acquire harmonically $\psi$ -convex function as follows:

$$
\begin{aligned}
& Q\left(\frac{a_{1}\left(a_{1}+\mathscr{F}_{\rho, \sigma}^{\lambda}\left(a_{2}-a_{1}\right)\right)}{a_{1}+t_{1} \mathscr{F}_{\rho, \sigma}^{\lambda}\left(a_{2}-a_{1}\right)}\right) \leq t_{1}^{s} Q\left(a_{1}\right)+\left(1-t_{1}\right)^{s} Q\left(a_{2}\right), \\
&\left.\cdot\left(\forall a_{1}, a_{2} \in \mathbb{H}_{\mathscr{F}}, t_{1} \in[0,1]\right), s \in(0,1]\right) .
\end{aligned}
$$

(ii) If we take $s=1$ and $\mathscr{F}_{\rho, \sigma}^{\lambda}\left(a_{2}-a_{1}\right)=a_{2}-a_{1}$, then we get Definition 3.1 in [13]

(iii) If we take $\mathscr{F}_{\rho, \sigma}^{\lambda}\left(a_{2}-a_{1}\right)=a_{2}-a_{1}$ along with $\widehat{\alpha}=s$ $=1$, then we get Definition in [37]

Recall the two special functions on Yang's fractal sets.

(1) Generalized beta function

$$
\begin{aligned}
\mathbb{B}_{\widehat{\alpha}}\left(z_{1}, z_{2}\right)= & \frac{1}{\Gamma(1+\widehat{\alpha})} \int_{0}^{1} t_{1}^{\widehat{\alpha}\left(z_{1}-1\right)}\left(1-t_{1}\right)^{\widehat{\alpha}\left(z_{2}-1\right)}\left(d t_{1}\right)^{\widehat{\alpha}} \\
& \cdot\left(z_{1}, z_{2}>0\right) .
\end{aligned}
$$


(2) Generalized hypergeometric function

$$
\begin{aligned}
{ }_{2} \bar{F}_{1}^{\widehat{\alpha}}\left(z_{1} ; z_{2} ; z_{3}, z\right)= & \frac{1}{\mathbb{B}_{\widehat{\alpha}}\left(z_{2}, z_{3}-z_{2}\right)} \frac{1}{\Gamma(1+\widehat{\alpha})} \int_{0}^{1} t_{1}^{\widehat{\alpha}\left(z_{1}-1\right)} \\
& \cdot\left(1-t_{1}\right)^{\widehat{\alpha}\left(z_{3}-z_{2}-1\right)}\left(1-z t_{1}\right)^{-\widehat{\alpha} z_{1}} \\
& \cdot\left(d t_{1}\right)^{\widehat{\alpha}}, 0<z_{2}<z_{3},|z|<1 .
\end{aligned}
$$

\section{Hermite-Hadamard Type Inequality}

In this section, we derive a novel version of HermiteHadamard inequalities for generalized harmonically $\psi$-convex functions, and this is the main motivation of this paper.

Theorem 13. For $s \in(0,1], \rho, \sigma>0, \lambda=\{\lambda(m)\}_{m=0}^{\infty} \quad a$ bounded sequence of real numbers and $Q: \mathscr{I}_{\mathscr{F}}=\left[a_{1}, a_{1}+\right.$ $\left.\mathscr{F}_{\rho, \sigma}^{\lambda}\left(a_{2}-a_{1}\right)\right] \subset \mathbb{R} \backslash\{0\} \mapsto \mathbb{R}^{\widehat{\alpha}}(0<\widehat{\alpha} \leq 1)$ be generalized harmonically $\psi-s$-convex function where $a_{1}, a_{1}+\mathscr{F}_{\rho, \sigma}^{\lambda}\left(a_{2}\right.$

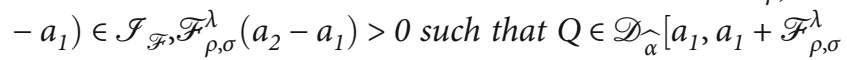
$\left.\left(a_{2}-a_{1}\right)\right]$ and $Q^{(\widehat{\alpha})} \in \mathbb{C}_{\widehat{\alpha}}\left[a_{1}, a_{1}+\mathscr{F}_{\rho, \sigma}^{\lambda}\left(a_{2}-a_{1}\right)\right]$. Then, the following inequalities hold:

$$
\begin{aligned}
& \frac{2^{(s-1) \widehat{\alpha}}}{\Gamma(1+\widehat{\alpha})} Q\left(\frac{2 a_{1}\left(a_{1}+\mathscr{F}_{\rho, \sigma}^{\lambda}\left(a_{2}-a_{1}\right)\right)}{\left(2 a_{1}+\mathscr{F}_{\rho, \sigma}^{\lambda}\left(a_{2}-a_{1}\right)\right)}\right) \\
& \quad \leq \frac{a_{1}^{\widehat{\alpha}}\left(a_{1}+\mathscr{F}_{\rho, \sigma}^{\lambda}\left(a_{2}-a_{1}\right)\right)^{\widehat{\alpha}}}{\left(\mathscr{F}_{\rho, \sigma}^{\lambda}\left(a_{2}-a_{1}\right)\right)^{\widehat{\alpha}}} \mathscr{F}_{a_{1}+\mathscr{F}_{\rho, \sigma}^{\lambda}\left(a_{2}-a_{1}\right)}^{(\widehat{\alpha}} \frac{Q(z)}{z^{2 \widehat{\alpha}}} \\
& \leq \frac{\Gamma(1+s \widehat{\alpha})}{\Gamma(1+(s+1) \widehat{\alpha})}\left[Q\left(a_{1}\right)+Q\left(a_{2}\right)\right] .
\end{aligned}
$$

Proof. Since $Q$ is generalized harmonically $\psi$-s-convex function with $t_{1}=1 / 2$, then

$Q\left(\frac{2 x\left(x+\mathscr{F}_{\rho, \sigma}^{\lambda}(y-x)\right)}{\left(2 x+\mathscr{F}_{\rho, \sigma}^{\lambda}(y-x)\right)}\right) \leq \frac{Q(x)+Q(y)}{2^{s \widehat{\alpha}}},\left(\forall x, y \in \mathscr{I}_{\mathscr{F}}, t_{1} \in[0,1]\right)$.

Let $\quad x=a_{1}\left(a_{1}+\mathscr{F}_{\rho, \sigma}^{\lambda}\left(a_{2}-a_{1}\right)\right) /\left(a_{1}+\left(1-t_{1}\right) \mathscr{F}_{\rho, \sigma}^{\lambda}\left(a_{2}-\right.\right.$ $\left.\left.a_{1}\right)\right)$ and $y=a_{1}\left(a_{1}+\mathscr{F}_{\rho, \sigma}^{\lambda}\left(a_{2}-a_{1}\right)\right) /\left(a_{1}+t_{1} \mathscr{F}_{\rho, \sigma}^{\lambda}\left(a_{2}-a_{1}\right)\right)$ in (25). Then, by local fractional integration over $(0,1)$, we have

$$
\begin{aligned}
& \frac{2^{\widehat{s \alpha}}}{\Gamma(1+\widehat{\alpha})} \int_{0}^{1} Q\left(\frac{2 a_{1}\left(a_{1}+\mathscr{F}_{\rho, \sigma}^{\lambda}\left(a_{2}-a_{1}\right)\right)}{\left(2 a_{1}+\mathscr{F}_{\rho, \sigma}^{\lambda}\left(a_{2}-a_{1}\right)\right)}\right)\left(d t_{1}\right)^{\widehat{\alpha}} \\
& \leq \frac{1}{\Gamma(1+\widehat{\alpha})} \int_{0}^{1}\left[Q\left(\frac{a_{1}\left(a_{1}+\mathscr{F}_{\rho, \sigma}^{\lambda}\left(a_{2}-a_{1}\right)\right)}{\left(a_{1}+\left(1-t_{1}\right) \mathscr{F}_{\rho, \sigma}^{\lambda}\left(a_{2}-a_{1}\right)\right)}\right)\right. \\
& \left.+Q\left(\frac{a_{1}\left(a_{1}+\mathscr{F}_{\rho, \sigma}^{\lambda}\left(a_{2}-a_{1}\right)\right)}{\left(a_{1}+t_{1} \mathscr{F}_{\rho, \sigma}^{\lambda}\left(a_{2}-a_{1}\right)\right)}\right)\right]\left(d t_{1}\right)^{\widehat{\alpha}}
\end{aligned}
$$

$$
\begin{aligned}
& =\frac{2^{\widehat{\alpha}} a_{1}^{\widehat{\alpha}}\left(a_{1}+\mathscr{F}_{\rho, \sigma}^{\lambda}\left(a_{2}-a_{1}\right)\right)^{\widehat{\alpha}}}{\left(\mathscr{F}_{\rho, \sigma}^{\lambda}\left(a_{2}-a_{1}\right)\right)^{\widehat{\alpha}}} \frac{1}{\Gamma(1+\widehat{\alpha})} \int_{a_{1}}^{a_{1}+\mathscr{F}_{p, \sigma}^{\lambda}\left(a_{2}-a_{1}\right)} \frac{Q(z)}{z^{2 \widehat{\alpha}}}(d z)^{\widehat{\alpha}} \\
& =\frac{2^{\widehat{\alpha}} a_{1}^{\widehat{\alpha}}\left(a_{1}+\mathscr{F}_{\rho, \sigma}^{\lambda}\left(a_{2}-a_{1}\right)\right)^{\widehat{\alpha}}}{\left(\mathscr{F}_{\rho, \sigma}^{\lambda}\left(a_{2}-a_{1}\right)\right)^{\widehat{\alpha}}} \mathscr{F}_{a_{1}+\mathscr{F}_{\rho, \sigma}^{\lambda}\left(a_{2}-a_{1}\right)}^{(\widehat{\alpha})} \frac{Q(z)}{z^{2 \widehat{\alpha}}} .
\end{aligned}
$$

Also, using the fact that

$$
\begin{gathered}
\frac{2^{\widehat{s \alpha}}}{\Gamma(1+\widehat{\alpha})} \int_{0}^{1} Q\left(\frac{2 a_{1}\left(a_{1}+\mathscr{F}_{\rho, \sigma}^{\lambda}\left(a_{2}-a_{1}\right)\right)}{\left(2 a_{1}+\mathscr{F}_{\rho, \sigma}^{\lambda}\left(a_{2}-a_{1}\right)\right)}\right)\left(d t_{1}\right)^{\widehat{\alpha}} \\
=\frac{2^{\widehat{s \alpha}}}{\Gamma(1+\widehat{\alpha})} Q\left(\frac{2 a_{1}\left(a_{1}+\mathscr{F}_{\rho, \sigma}^{\lambda}\left(a_{2}-a_{1}\right)\right)}{\left(2 a_{1}+\mathscr{F}_{\rho, \sigma}^{\lambda}\left(a_{2}-a_{1}\right)\right)}\right),
\end{gathered}
$$

we have

$$
\begin{aligned}
& \frac{2^{(s-1) \widehat{\alpha}}}{\Gamma(1+\widehat{\alpha})} Q\left(\frac{2 a_{1}\left(a_{1}+\mathscr{F}_{\rho, \sigma}^{\lambda}\left(a_{2}-a_{1}\right)\right)}{\left(2 a_{1}+\mathscr{F}_{\rho, \sigma}^{\lambda}\left(a_{2}-a_{1}\right)\right)}\right) \\
& \leq \frac{a_{1}^{\widehat{\alpha}}\left(a_{1}+\mathscr{F}_{\rho, \sigma}^{\lambda}\left(a_{2}-a_{1}\right)\right)^{\widehat{\alpha}}}{\left(\mathscr{F}_{\rho, \sigma}^{\lambda}\left(a_{2}-a_{1}\right)\right)^{\widehat{\alpha}}} \mathscr{F}_{a_{1}+\mathscr{F}_{\rho, \sigma}^{\lambda}\left(a_{2}-a_{1}\right)}^{(\widehat{\alpha})} \frac{Q(z)}{z^{2 \widehat{\alpha}}} .
\end{aligned}
$$

For the proof of other inequality in (73), noting that $Q$ is a generalized harmonically $\psi$-s-convex mapping, for $t_{1} \in[0,1]$, we have

$$
\begin{gathered}
Q\left(\frac{a_{1}\left(a_{1}+\mathscr{F}_{\rho, \sigma}^{\lambda}\left(a_{2}-a_{1}\right)\right)}{\left(a_{1}+\left(1-t_{1}\right) \mathscr{F}_{\rho, \sigma}^{\lambda}\left(a_{2}-a_{1}\right)\right)}\right) \\
+Q\left(\frac{a_{1}\left(a_{1}+\mathscr{F}_{\rho, \sigma}^{\lambda}\left(a_{2}-a_{1}\right)\right)}{\left(a_{1}+t_{1} \mathscr{F}_{\rho, \sigma}^{\lambda}\left(a_{2}-a_{1}\right)\right)}\right) \\
\leq\left[t_{1}^{s \widehat{\alpha}}+\left(1-t_{1}\right)^{\widehat{s \alpha}}\right]\left[Q\left(a_{1}\right)+Q\left(a_{2}\right)\right] .
\end{gathered}
$$

Again, using local fractional integration for above inequality with respect to $t_{1}$ over $(0,1)$ yields

$$
\begin{aligned}
& \frac{a_{1}^{\widehat{\alpha}}\left(a_{1}+\mathscr{F}_{\rho, \sigma}^{\lambda}\left(a_{2}-a_{1}\right)\right)^{\widehat{\alpha}}}{\left(\mathscr{F}_{\rho, \sigma}^{\lambda}\left(a_{2}-a_{1}\right)\right)^{\widehat{\alpha}}} \mathscr{F}_{a_{1}+\mathscr{F}_{\rho, \sigma}^{\lambda}\left(a_{2}-a_{1}\right)}^{(\widehat{\alpha})} \frac{Q(z)}{z^{2 \widehat{\alpha}}} \\
& \leq \frac{\Gamma(1+s \widehat{\alpha})}{\Gamma(1+(s+1) \widehat{\alpha})}\left[Q\left(a_{1}\right)+Q\left(a_{2}\right)\right] .
\end{aligned}
$$

Combining (28) and (30), we get the inequality (24). Hence, the proof is completed. 
Certain noteworthy cases of Theorem 13 are established as follows:

(i) Choosing $\widehat{\alpha}=1$, then we attain a new consequence for harmonically $\psi$-s-convex function

Corollary 14. For $\rho, \sigma>0, \lambda=\{\lambda(m)\}_{m=0}^{\infty}$ a bounded sequence of real numbers and $Q: \mathscr{I}_{\mathscr{F}}=\left[a_{1}, a_{1}+\mathscr{F}_{\rho, \sigma}^{\lambda}\left(a_{2}-\right.\right.$ $\left.\left.a_{1}\right)\right] \subset \mathbb{R} \backslash\{0\} \mapsto \mathbb{R}$ be harmonically $\psi$ - s-convex function where $a_{1}, a_{1}+\mathscr{F}_{\rho, \sigma}^{\lambda}\left(a_{2}-a_{1}\right) \in \mathscr{I}_{\mathscr{F}, \mathscr{F}_{\rho, \sigma}^{\lambda}}^{\lambda}\left(a_{2}-a_{1}\right)>0$ such that $Q^{\prime} \in L_{1}\left(\left[a_{1}, a_{1}+\mathscr{F}_{\rho, \sigma}^{\lambda}\left(a_{2}-a_{1}\right)\right]\right)$. Then, the following inequalities hold:

$$
\begin{aligned}
& 2^{s-1} Q\left(\frac{2 a_{1}\left(a_{1}+\mathscr{F}_{\rho, \sigma}^{\lambda}\left(a_{2}-a_{1}\right)\right)}{\left(2 a_{1}+\mathscr{F}_{\rho, \sigma}^{\lambda}\left(a_{2}-a_{1}\right)\right)}\right) \\
& \quad \leq \frac{a_{1}\left(a_{1}+\mathscr{F}_{\rho, \sigma}^{\lambda}\left(a_{2}-a_{1}\right)\right)}{\left(\mathscr{F}_{\rho, \sigma}^{\lambda}\left(a_{2}-a_{1}\right)\right)} \int_{a_{1}}^{a_{1}+\mathscr{F}_{\rho, \sigma}^{\lambda}\left(a_{2}-a_{1}\right)} \frac{Q(z)}{z^{2}} d z \\
& \leq \frac{Q\left(a_{1}\right)+Q\left(a_{2}\right)}{s+1} .
\end{aligned}
$$

Remark 15. In view of Theorem 13, we have that

(i) Letting $s=1$ and $\mathscr{F}_{\rho, \sigma}^{\lambda}\left(a_{2}-a_{1}\right)=a_{2}-a_{1}$, then we get Theorem 4.1 of [13].

(ii) Letting $\widehat{\alpha}=1$ and $\mathscr{F}_{\rho, \sigma}^{\lambda}\left(a_{2}-a_{1}\right)=a_{2}-a_{1}$, then we get Theorem 3 of [39].

Lemma 16. For $\rho, \sigma>0, \lambda=\{\lambda(m)\}_{m=0}^{\infty}$ a bounded sequence of real numbers and $Q: \mathscr{I}_{\mathscr{F}}^{\circ}=\left[a_{1}, a_{1}+\mathscr{F}_{\rho, \sigma}^{\lambda}\left(a_{2}-a_{1}\right)\right] \subset \mathbb{R} \backslash$ $\{0\} \mapsto \mathbb{R}^{\widehat{\alpha}}(0<\widehat{\alpha} \leq 1)\left(\mathscr{I}_{\mathscr{F}}^{\circ}\right.$ is the interior of $\left.\mathscr{I}_{\mathscr{F}}\right)$ be a generalized harmonically $\psi$-convex function, where $a_{1}, a_{1}+\mathscr{F}_{\rho, \sigma}^{\lambda}$ $\left(a_{2}-a_{1}\right) \in \mathscr{I}_{\mathscr{F}}^{\circ}, \mathscr{F}_{\rho, \sigma}^{\lambda}\left(a_{2}-a_{1}\right)>0$ such that $Q \in \mathscr{D}_{\hat{\alpha}}\left[a_{1}, a_{1}+\right.$ $\left.\mathscr{F}_{\rho, \sigma}^{\lambda}\left(a_{2}-a_{1}\right)\right]$ and $Q^{(\widehat{\alpha})} \in \mathbb{C}_{\widehat{\alpha}}\left[a_{1}, a_{1}+\mathscr{F}_{\rho, \sigma}^{\lambda}\left(a_{2}-a_{1}\right)\right]$. Then, the identity

$$
\begin{aligned}
\Omega_{Q}\left(a_{1}, a_{1}+\mathscr{F}_{\rho, \sigma}^{\lambda}\left(a_{2}-a_{1}\right) ; \omega, \widehat{\alpha}\right) \\
:=\frac{\left(\mathscr{F}_{\rho, \sigma}^{\lambda}\left(a_{2}-a_{1}\right)\right)^{\widehat{\alpha}}}{\widehat{a_{1}^{\widehat{\alpha}}}\left(a_{1}+\mathscr{F}_{\rho, \sigma}^{\lambda}\left(a_{2}-a_{1}\right)\right)^{\widehat{\alpha}}} \frac{1}{\Gamma(1+\widehat{\alpha})} \int_{0}^{1-\omega} t_{1}^{\widehat{\alpha}} \\
\quad \times\left(\frac{a_{1}\left(a_{1}+\mathscr{F}_{\rho, \sigma}^{\lambda}\left(a_{2}-a_{1}\right)\right)}{a_{1}+t_{1} \mathscr{F}_{\rho, \sigma}^{\lambda}\left(a_{2}-a_{1}\right)}\right)^{2 \widehat{\alpha}} Q^{(\widehat{\alpha})} \\
\quad \times\left(\frac{a_{1}\left(a_{1}+\mathscr{F}_{\rho, \sigma}^{\lambda}\left(a_{2}-a_{1}\right)\right)}{a_{1}+t_{1} \mathscr{F}_{\rho, \sigma}^{\lambda}\left(a_{2}-a_{1}\right)}\right)\left(d t_{1}\right)^{\widehat{\alpha}}
\end{aligned}
$$

$$
\begin{aligned}
& +\frac{1}{\Gamma(1+\widehat{\alpha})} \int_{1-\omega}^{1}\left(t_{1}-1\right)^{\widehat{\alpha}}\left(\frac{a_{1}\left(a_{1}+\mathscr{F}_{\rho, \sigma}^{\lambda}\left(a_{2}-a_{1}\right)\right)}{a_{1}+t_{1} \mathscr{F}_{\rho, \sigma}^{\lambda}\left(a_{2}-a_{1}\right)}\right)^{2 \widehat{\alpha}} Q^{(\widehat{a}} \\
& \left.\times\left(\frac{a_{1}\left(a_{1}+\mathscr{F}_{\rho, \sigma}^{\lambda}\left(a_{2}-a_{1}\right)\right)}{a_{1}+t_{1} \mathscr{F}_{\rho, \sigma}^{\lambda}\left(a_{2}-a_{1}\right)}\right)\left(d t_{1}\right)^{\widehat{\alpha}}\right]
\end{aligned}
$$

where

$$
\begin{aligned}
\Omega_{Q}\left(a_{1}, a_{1}+\mathscr{F}_{\rho, \sigma}^{\lambda}\left(a_{2}-a_{1}\right) ; \omega, \widehat{\alpha}\right) \\
:=(1-\omega)^{\widehat{\alpha}} Q\left(\frac{a_{1}\left(a_{1}+\mathscr{F}_{\rho, \sigma}^{\lambda}\left(a_{2}-a_{1}\right)\right)}{a_{1}+t_{1} \mathscr{F}_{\rho, \sigma}^{\lambda}\left(a_{2}-a_{1}\right)}\right) \\
+\omega^{\widehat{\alpha}} Q\left(\frac{a_{1}\left(a_{1}+\mathscr{F}_{\rho, \sigma}^{\lambda}\left(a_{2}-a_{1}\right)\right)}{a_{1}+t_{1} \mathscr{F}_{\rho, \sigma}^{\lambda}\left(a_{2}-a_{1}\right)}\right) \\
-\left(\frac{a_{1}\left(a_{1}+\mathscr{F}_{\rho, \sigma}^{\lambda}\left(a_{2}-a_{1}\right)\right)}{\mathscr{F}_{\rho, \sigma}^{\lambda}\left(a_{2}-a_{1}\right)}\right) \\
\quad \cdot \Gamma(1+\widehat{\alpha})_{a_{1}} \mathcal{F}_{a_{1}+\mathscr{F}_{\rho, \sigma}^{\lambda}\left(a_{2}-a_{1}\right)}^{(\widehat{\alpha})} \frac{Q(z)}{z^{2 \alpha}},
\end{aligned}
$$

holds for $\omega \in[0,1]$ and $\widehat{\alpha} \in(0,1]$.

Proof. By local fractional integration by parts, we have

$$
\begin{aligned}
\mathscr{J}_{1}= & \frac{1}{\Gamma(1+\widehat{\alpha})} \int_{0}^{1-\omega} \widehat{t_{1}^{\alpha}}\left(\frac{a_{1}\left(a_{1}+\mathscr{F}_{\rho, \sigma}^{\lambda}\left(a_{2}-a_{1}\right)\right)}{a_{1}+t_{1} \mathscr{F}_{\rho, \sigma}^{\lambda}\left(a_{2}-a_{1}\right)}\right)^{2 \widehat{\alpha}} Q^{(\widehat{\alpha})} \\
& \cdot\left(\frac{a_{1}\left(a_{1}+\mathscr{F}_{\rho, \sigma}^{\lambda}\left(a_{2}-a_{1}\right)\right)}{a_{1}+t_{1} \mathscr{F}_{\rho, \sigma}^{\lambda}\left(a_{2}-a_{1}\right)}\right)\left(d t_{1}\right)^{\widehat{\alpha}} \\
= & \left.t_{1}^{\alpha} Q\left(\frac{a_{1}\left(a_{1}+\mathscr{F}_{\rho, \sigma}^{\lambda}\left(a_{2}-a_{1}\right)\right)}{a_{1}+t_{1} \mathscr{F}_{\rho, \sigma}^{\lambda}\left(a_{2}-a_{1}\right)}\right)\right|_{0} ^{1-\omega}-\frac{\Gamma(1+\widehat{\alpha})}{\Gamma(1+\widehat{\alpha})} \int_{0}^{1-\omega} Q \\
& \cdot\left(\frac{a_{1}\left(a_{1}+\mathscr{F}_{\rho, \sigma}^{\lambda}\left(a_{2}-a_{1}\right)\right)}{a_{1}+t_{1} \mathscr{F}_{\rho, \sigma}^{\lambda}\left(a_{2}-a_{1}\right)}\right)\left(d t_{1}\right)^{\alpha} \\
= & (1-\omega)^{\widehat{\alpha}} Q\left(\frac{a_{1}\left(a_{1}+\mathscr{F}_{\rho, \sigma}^{\lambda}\left(a_{2}-a_{1}\right)\right)}{a_{1}+t_{1} \mathscr{F}_{\rho, \sigma}^{\lambda}\left(a_{2}-a_{1}\right)}\right)-\frac{\Gamma(1+\widehat{\alpha})}{\Gamma(1+\widehat{\alpha})} \int_{0}^{1-\omega} Q \\
& \cdot\left(\frac{a_{1}\left(a_{1}+\mathscr{F}_{\rho, \sigma}^{\lambda}\left(a_{2}-a_{1}\right)\right)}{a_{1}+t_{1} \mathscr{F}_{\rho, \sigma}^{\lambda}\left(a_{2}-a_{1}\right)}\right)\left(d t_{1}\right)^{\alpha} .
\end{aligned}
$$


Journal of Function Spaces

7

Setting $\quad z=a_{1}\left(a_{1}+\mathscr{F}_{\rho, \sigma}^{\lambda}\left(a_{2}-a_{1}\right)\right) / a_{1}+t_{1} \mathscr{F}_{\rho, \sigma}^{\lambda}\left(a_{2}-a_{1}\right)$, then

$$
\begin{aligned}
& \mathscr{J}_{1}=(1-\omega)^{\widehat{\alpha}} Q\left(\frac{a_{1}\left(a_{1}+\mathscr{F}_{\rho, \sigma}^{\lambda}\left(a_{2}-a_{1}\right)\right)}{a_{1}+t_{1} \mathscr{F}_{\rho, \sigma}^{\lambda}\left(a_{2}-a_{1}\right)}\right) \\
& -\left(\frac{a_{1}\left(a_{1}+\mathscr{F}_{\rho, \sigma}^{\lambda}\left(a_{2}-a_{1}\right)\right)}{\mathscr{F}_{\rho, \sigma}^{\lambda}\left(a_{2}-a_{1}\right)}\right)^{\widehat{\alpha}} \frac{\Gamma(1+\widehat{\alpha})}{\Gamma(1+\widehat{\alpha})} \\
& \cdot \int_{a_{1}}^{\frac{a_{1}\left(a_{1}+\mathcal{F}_{\rho, \sigma}^{\lambda}\left(a_{2}-a_{1}\right)\right)}{a_{1}+\omega \mathcal{F}_{\rho, \sigma}^{\prime}\left(a_{2}-a_{1}\right)}} \frac{Q(z)}{z^{2 \widehat{\alpha}}}(d z)^{\widehat{\alpha}} \\
& =(1-\omega){ }^{\widehat{\alpha}} Q\left(\frac{a_{1}\left(a_{1}+\mathscr{F}_{\rho, \sigma}^{\lambda}\left(a_{2}-a_{1}\right)\right)}{a_{1}+t_{1} \mathscr{F}_{\rho, \sigma}^{\lambda}\left(a_{2}-a_{1}\right)}\right) \\
& -\left(\frac{a_{1}\left(a_{1}+\mathscr{F}_{\rho, \sigma}^{\lambda}\left(a_{2}-a_{1}\right)\right)}{\mathscr{F}_{\rho, \sigma}^{\lambda}\left(a_{2}-a_{1}\right)}\right)^{\widehat{\alpha}} \Gamma \\
& \cdot(1+\widehat{\alpha})_{a_{1}} \mathscr{J}_{a_{1}\left(a_{1}+\mathscr{F}_{\rho, \sigma}^{\lambda}\left(a_{2}-a_{1}\right)\right) / a_{1}+\omega \mathscr{F}_{\rho, \sigma}^{\lambda}\left(a_{2}-a_{1}\right)}^{(\widehat{\alpha})} \frac{Q(z)}{z^{2 \widehat{\alpha}}} .
\end{aligned}
$$

Analogously, we have

$$
\begin{aligned}
& \mathscr{J}_{2}=\frac{1}{\Gamma(1+\widehat{\alpha})} \int_{1-\omega}^{1}\left(t_{1}-1\right)^{\widehat{\alpha}}\left(\frac{a_{1}\left(a_{1}+\mathscr{F}_{\rho, \sigma}^{\lambda}\left(a_{2}-a_{1}\right)\right)}{a_{1}+t_{1} \mathscr{F}_{\rho, \sigma}^{\lambda}\left(a_{2}-a_{1}\right)}\right)^{2 \widehat{\alpha}} \\
& \cdot Q^{(\widehat{\alpha})}\left(\frac{a_{1}\left(a_{1}+\mathscr{F}_{\rho, \sigma}^{\lambda}\left(a_{2}-a_{1}\right)\right)}{a_{1}+t_{1} \mathscr{F}_{\rho, \sigma}^{\lambda}\left(a_{2}-a_{1}\right)}\right)\left(d t_{1}\right)^{\widehat{\alpha}} \\
& =\omega^{\widehat{\alpha}} Q\left(\frac{a_{1}\left(a_{1}+\mathscr{F}_{\rho, \sigma}^{\lambda}\left(a_{2}-a_{1}\right)\right)}{a_{1}+t_{1} \mathscr{F}_{\rho, \sigma}^{\lambda}\left(a_{2}-a_{1}\right)}\right) \\
& -\left(\frac{a_{1}\left(a_{1}+\mathscr{F}_{\rho, \sigma}^{\lambda}\left(a_{2}-a_{1}\right)\right)}{\mathscr{F}_{\rho, \sigma}^{\lambda}\left(a_{2}-a_{1}\right)}\right)^{\widehat{\alpha}} \Gamma \\
& \cdot(1+\widehat{\alpha})_{a_{1}\left(a_{1}+\mathscr{F}_{\rho, \sigma}^{\lambda}\left(a_{2}-a_{1}\right)\right) / a_{1}+\omega \mathscr{F}_{\rho, \sigma}^{\lambda}\left(a_{2}-a_{1}\right)} \mathscr{J}_{a_{1}+\mathscr{F}_{p, \sigma}^{\lambda}\left(a_{2}-a_{1}\right)}^{(\widehat{\alpha})} \frac{Q(z)}{z^{2 \widehat{\alpha}}} .
\end{aligned}
$$

Adding $\mathscr{J}_{1}$ and $\mathscr{J}_{2}$, we obtain the required result. This completes the proof of Lemma 16.

Theorem 17. Assume that the hypothesis of Lemma 16 are satisfied. If $\left|Q^{(\widehat{\alpha})}\right|^{q_{1}}$ is a generalized harmonically $\psi$ - $s$-convex function on $\mathscr{I}_{\mathscr{F}}$ for $p, q>1, p^{-1}+q^{-1}=1$. Then, the following inequality for local fractional integrals holds

$$
\begin{aligned}
& \left|\Omega_{Q}\left(a_{1}, a_{1}+\mathscr{F}_{\rho, \sigma}^{\lambda}\left(a_{2}-a_{1}\right) ; \omega, \widehat{\alpha}\right)\right| \\
& \leq \frac{\left(\mathscr{F}_{\rho, \sigma}^{\lambda}\left(a_{2}-a_{1}\right)\right)^{\widehat{\alpha}}}{a_{1}^{\widehat{\alpha}}\left(a_{1}+\mathscr{F}_{\rho, \sigma}^{\lambda}\left(a_{2}-a_{1}\right)\right)^{\widehat{\alpha}}}\left(\frac{\Gamma(1+\widehat{\alpha})}{\Gamma(1+2 \widehat{\alpha})}\right)^{1-1 / q} \\
& \times\left[\left\{( 1 - \omega ) ^ { 2 ( 1 - 1 / q ) } \widehat { \alpha } \left[\Lambda_{1}^{\widehat{\alpha}}\left(q, \omega, a_{1}, a_{2} ; s\right)\left|Q^{(\widehat{\alpha})}\left(a_{1}\right)\right|^{q}\right.\right.\right. \\
& \left.\left.+\Lambda_{2}^{\widehat{\alpha}}\left(q, \omega, a_{1}, a_{2} ; s\right)\left|Q^{(\widehat{\alpha})}\left(a_{2}\right)\right|^{q}\right]\right\}^{1 / q} \\
& +\left\{( \omega ) ^ { 2 ( 1 - 1 / q ) \widehat { \alpha } } \left[\Lambda_{3}^{\widehat{\alpha}}\left(q, \omega, a_{1}, a_{2} ; s\right)\left|Q^{(\widehat{\alpha})}\left(a_{1}\right)\right|^{q}\right.\right. \\
& \left.\left.\left.+\Lambda_{4}^{\widehat{\alpha}}\left(q, \omega, a_{1}, a_{2} ; s\right)\left|Q^{(\widehat{\alpha})}\left(a_{2}\right)\right|^{q}\right]\right\}^{1 / q}\right] \text {, }
\end{aligned}
$$

where

$$
\begin{aligned}
\Lambda_{1}^{\widehat{\alpha}}\left(q, \omega, a_{1}, a_{2} ; s\right) & \\
:= & (1-\omega)^{(s+2) \widehat{\alpha}}\left(a_{1}+\mathscr{F}_{\rho, \sigma}^{\lambda}\left(a_{2}-a_{1}\right)\right)^{2 q \widehat{\alpha}} \times \frac{\Gamma(1+(s+1) \widehat{\alpha})}{\Gamma(1+(s+2) \widehat{\alpha})_{2}} \bar{F}_{1}^{\widehat{\alpha}} \\
& \times\left(2 q, s+2 ; s+3 ; \frac{(\omega-1) \mathscr{F}_{\rho, \sigma}^{\lambda}\left(a_{2}-a_{1}\right)}{a_{1}}\right),
\end{aligned}
$$

$$
\begin{aligned}
& \Lambda_{2}^{\widehat{\alpha}}\left(q, \omega, a_{1}, a_{2} ; s\right) \\
& :=(1-\omega)^{2 \widehat{\alpha}}\left(a_{1}+\mathscr{F}_{\rho, \sigma}^{\lambda}\left(a_{2}-a_{1}\right)\right)^{2 q \widehat{\alpha}} \frac{\Gamma(1+\widehat{\alpha})}{\Gamma(1+2 \widehat{\alpha})} \times{ }_{2} \bar{F}_{1}^{\widehat{\alpha}} \\
& \quad \times\left(2 q, 2 ; 3 ; \frac{(\omega-1) \mathscr{F}_{\rho, \sigma}^{\lambda}\left(a_{2}-a_{1}\right)}{a_{1}}\right)-\Lambda_{1}^{\widehat{\alpha}}\left(q, \omega, a_{1}, a_{2} ; s\right),
\end{aligned}
$$

$\Lambda_{3}^{\widehat{\alpha}}\left(q, \omega, a_{1}, a_{2} ; s\right)$

$$
\begin{aligned}
:= & \left(a_{1}+\mathscr{F}_{\rho, \sigma}^{\lambda}\left(a_{2}-a_{1}\right)\right)^{2 q \widehat{\alpha}}\left[\frac{\Gamma(1+s \widehat{\alpha})}{\Gamma(1+(s+1) \widehat{\alpha})_{2}} \bar{F}_{1}^{\widehat{\alpha}}\right. \\
& \times\left(2 q, s+1 ; s+2 ; \frac{(\omega-1) \mathscr{F}_{\rho, \sigma}^{\lambda}\left(a_{2}-a_{1}\right)}{a_{1}}\right) \\
& -\frac{\Gamma(1+(s+2) \widehat{\alpha})}{\Gamma(1+(s+3) \widehat{\alpha})_{2}} \bar{F}_{1}^{\widehat{\alpha}}\left(2 q, s+2 ; s+3 ; \frac{(\omega-1) \mathscr{F}_{\rho, \sigma}^{\lambda}\left(a_{2}-a_{1}\right)}{a_{1}}\right) \\
& -(1-\omega)^{(s+1) \widehat{\alpha}} \frac{\Gamma(1+s \widehat{\alpha})}{\Gamma(1+(s+1) \widehat{\alpha})} \times{ }_{2} \bar{F}_{1}^{\widehat{\alpha}} \\
& \left.\times\left(2 q,(s+1) ;(s+2) ; \frac{(\omega-1) \mathscr{F}_{\rho, \sigma}^{\lambda}\left(a_{2}-a_{1}\right)}{a_{1}}\right)\right] \\
& +\Lambda_{1}^{\widehat{\alpha}}\left(q, \omega, a_{1}, a_{2} ; s\right),
\end{aligned}
$$

$$
\begin{aligned}
\Lambda_{4}^{\widehat{\alpha}}\left(q, \omega, a_{1}, a_{2} ; s\right) \\
:=\left(a_{1}+\mathscr{F}_{\rho, \sigma}^{\lambda}\left(a_{2}-a_{1}\right)\right)^{2 q \widehat{\alpha}}\left[\frac{\Gamma(1+(s+2) \widehat{\alpha})}{\Gamma(1+(s+3) \widehat{\alpha})_{2}} \bar{F}_{1}^{\widehat{\alpha}}\right. \\
\quad \times\left(2 q, 1 ; s+3 ; \frac{(\omega-1) \mathscr{F}_{\rho, \sigma}^{\lambda}\left(a_{2}-a_{1}\right)}{a_{1}}\right)
\end{aligned}
$$


8

Journal of Function Spaces

$$
\begin{aligned}
& -(1-\omega)^{\widehat{\alpha}} \Gamma(1+\widehat{\alpha})_{2} \bar{F}_{1}^{\widehat{\alpha}}\left(2 q, 1 ; 2 ; \frac{(\omega-1) \mathscr{F}_{\rho, \sigma}^{\lambda}\left(a_{2}-a_{1}\right)}{a_{1}}\right) \\
& +(1-\omega)^{(s+2) \widehat{\alpha}} \frac{\Gamma(1+(s+2) \widehat{\alpha})}{\Gamma(1+(s+3) \widehat{\alpha})_{2}} \bar{F}_{1}^{\widehat{\alpha}} \\
& \left.\times\left(2 q, s+2 ; s+3 ; \frac{(\omega-1) \mathscr{F}_{\rho, \sigma}^{\lambda}\left(a_{2}-a_{1}\right)}{a_{1}}\right)\right]
\end{aligned}
$$

Proof. By means of Lemma 16, the generalized power mean inequality and the generalized harmonically $\psi-s$ -convexity of $\left|Q^{(\widehat{\alpha})}\right|^{q}$, we have

$$
\begin{aligned}
& \left|\Omega_{Q}\left(a_{1}, a_{1}+\mathscr{F}_{\rho, \sigma}^{\lambda}\left(a_{2}-a_{1}\right) ; \omega, \widehat{\alpha}\right)\right| \\
& \leq \frac{\left(\mathscr{F}_{\rho, \sigma}^{\lambda}\left(a_{2}-a_{1}\right)\right)^{\widehat{\alpha}}}{a_{1}^{\widehat{\alpha}}\left(a_{1}+\mathscr{F}_{\rho, \sigma}^{\lambda}\left(a_{2}-a_{1}\right)\right)^{\widehat{\alpha}}}\left[\frac{1}{\Gamma(1+\widehat{\alpha})} \int_{0}^{1-\omega} t_{1}^{\widehat{\alpha}}\right. \\
& \times\left(\frac{a_{1}\left(a_{1}+\mathscr{F}_{\rho, \sigma}^{\lambda}\left(a_{2}-a_{1}\right)\right)}{a_{1}+t_{1} \mathscr{F}_{\rho, \sigma}^{\lambda}\left(a_{2}-a_{1}\right)}\right)^{2 \widehat{\alpha}} \\
& \times\left|Q^{(\widehat{\alpha})}\left(\frac{a_{1}\left(a_{1}+\mathscr{F}_{\rho, \sigma}^{\lambda}\left(a_{2}-a_{1}\right)\right)}{a_{1}+t_{1} \mathscr{F}_{\rho, \sigma}^{\lambda}\left(a_{2}-a_{1}\right)}\right)\right|\left(d t_{1}\right)^{\widehat{\alpha}} \\
& +\frac{1}{\Gamma(1+\widehat{\alpha})} \int_{1-\omega}^{1}\left(t_{1}-1\right)^{\widehat{\alpha}}\left(\frac{a_{1}\left(a_{1}+\mathscr{F}_{\rho, \sigma}^{\lambda}\left(a_{2}-a_{1}\right)\right)}{a_{1}+t_{1} \mathscr{F}_{\rho, \sigma}^{\lambda}\left(a_{2}-a_{1}\right)}\right)^{2 \widehat{\alpha}} \\
& \left.\times\left|Q^{(\widehat{\alpha})}\left(\frac{a_{1}\left(a_{1}+\mathscr{F}_{\rho, \sigma}^{\lambda}\left(a_{2}-a_{1}\right)\right)}{a_{1}+t_{1} \mathscr{F}_{\rho, \sigma}^{\lambda}\left(a_{2}-a_{1}\right)}\right)\right|\left(d t_{1}\right)^{\widehat{\alpha}}\right] \\
& \leq \frac{\left(\mathscr{F}_{\rho, \sigma}^{\lambda}\left(a_{2}-a_{1}\right)\right)^{\widehat{\alpha}}}{a_{1}^{\widehat{\alpha}}\left(a_{1}+\mathscr{F}_{\rho, \sigma}^{\lambda}\left(a_{2}-a_{1}\right)\right)^{\widehat{\alpha}}}\left[\left(\frac{1}{\Gamma(1+\widehat{\alpha})} \int_{0}^{1-\omega} t_{1}^{\widehat{\alpha}}\left(d t_{1}\right)^{\widehat{\alpha}}\right)^{1-1 / q}\right. \\
& \times\left(\frac{1}{\Gamma(1+\widehat{\alpha})} \int_{0}^{1-\omega} t_{1}^{\widehat{\alpha}}\left(\frac{a_{1}\left(a_{1}+\mathscr{F}_{\rho, \sigma}^{\lambda}\left(a_{2}-a_{1}\right)\right)}{a_{1}+t_{1} \mathscr{F}_{\rho, \sigma}^{\lambda}\left(a_{2}-a_{1}\right)}\right)^{2 q \widehat{\alpha}}\right. \\
& \times\left.\left|Q^{(\widehat{\alpha})}\left(\frac{a_{1}\left(a_{1}+\mathscr{F}_{\rho, \sigma}^{\lambda}\left(a_{2}-a_{1}\right)\right)}{a_{1}+t_{1} \mathscr{F}_{\rho, \sigma}^{\lambda}\left(a_{2}-a_{1}\right)}\right)\right|^{q}\left(d t_{1}\right)^{\widehat{\alpha}}\right|^{1 / q} \\
& +\left(\frac{1}{\Gamma(1+\widehat{\alpha})} \int_{1-\omega}^{1}\left(1-t_{1}\right)^{\widehat{\alpha}}\left(d t_{1}\right)^{\widehat{\alpha}}\right)^{1-1 / q} \\
& \times\left(\frac{1}{\Gamma(1+\widehat{\alpha})} \int_{1-\omega}^{1}\left(1-t_{1}\right)^{\widehat{\alpha}}\left(\frac{a_{1}\left(a_{1}+\mathscr{F}_{\rho, \sigma}^{\lambda}\left(a_{2}-a_{1}\right)\right)}{a_{1}+t_{1} \mathscr{F}_{\rho, \sigma}^{\lambda}\left(a_{2}-a_{1}\right)}\right)^{2 \hat{q \alpha}}\right. \\
& \left.\times\left|Q^{(\widehat{\alpha})}\left(\frac{a_{1}\left(a_{1}+\mathscr{F}_{\rho, \sigma}^{\lambda}\left(a_{2}-a_{1}\right)\right)}{a_{1}+t_{1} \mathscr{F}_{\rho, \sigma}^{\lambda}\left(a_{2}-a_{1}\right)}\right)\right|^{q}\left(d t_{1}\right)^{\widehat{\alpha}}\right)^{1 / q}
\end{aligned}
$$

$$
\begin{aligned}
& \left.\leq \frac{\left(\mathscr{F}_{\rho, \sigma}^{\lambda}\left(a_{2}-a_{1}\right)\right)^{\widehat{\alpha}}}{a_{1}^{\widehat{\alpha}}\left(a_{1}+\mathscr{F}_{\rho, \sigma}^{\lambda}\left(a_{2}-a_{1}\right)\right)^{\widehat{\alpha}}}\right]\left[\left(\frac{1}{\Gamma(1+\widehat{\alpha})} \int_{0}^{1-\omega} t_{1}^{\widehat{\alpha}}\left(d t_{1}\right)^{\widehat{\alpha}}\right)^{1-1 / q}\right. \\
& \times\left(\frac{1}{\Gamma(1+\widehat{\alpha})} \int_{0}^{1-\omega}\left(\frac{a_{1}\left(a_{1}+\mathscr{F}_{\rho, \sigma}^{\lambda}\left(a_{2}-a_{1}\right)\right)}{a_{1}+t_{1} \mathscr{F}_{\rho, \sigma}^{\lambda}\left(a_{2}-a_{1}\right)}\right)^{2 q \widehat{\alpha}}\right. \\
& \times\left[t_{1}^{(s+1) \widehat{\alpha}}\left|Q^{(\widehat{\alpha})}\left(a_{1}\right)\right|^{q}+t_{1}^{\widehat{\alpha}}\left(1-t_{1}\right)^{\widehat{s \alpha}}\right. \\
& \left.\left.\times\left|Q^{(\widehat{\alpha})}\left(a_{2}\right)\right|^{q}\right]\left(d t_{1}\right)^{\widehat{\alpha}}\right)^{1 / q}+\left(\frac{1}{\Gamma(1+\widehat{\alpha})} \int_{1-\omega}^{1}\right. \\
& \left.\times\left(1-t_{1}\right)^{\widehat{\alpha}}\left(d t_{1}\right)^{\widehat{\alpha}}\right)^{1-1 / q} \times\left(\frac{1}{\Gamma(1+\widehat{\alpha})} \int_{1-\omega}^{1}\right. \\
& \times\left(\frac{a_{1}\left(a_{1}+\mathscr{F}_{\rho, \sigma}^{\lambda}\left(a_{2}-a_{1}\right)\right)}{a_{1}+t_{1} \mathscr{F}_{\rho, \sigma}^{\lambda}\left(a_{2}-a_{1}\right)}\right)^{2 q \widehat{\alpha}}\left[\left(1-t_{1}\right)^{\widehat{\alpha}} t_{1}^{s \widehat{\alpha}}\left|Q^{(\widehat{\alpha})}\left(a_{1}\right)\right|^{q}\right. \\
& \left.\left.\left.+\left(1-t_{1}\right)^{(s+1) \widehat{\alpha}}\left|Q^{(\widehat{\alpha})}\left(a_{2}\right)\right|^{q}\right]\left(d t_{1}\right)^{\widehat{\alpha}}\right)^{1 / q}\right] \text {. }
\end{aligned}
$$

Employing Lemma 7, we have that

$$
\begin{aligned}
& \frac{1}{\Gamma(1+\widehat{\alpha})} \int_{0}^{1-\omega} t_{1}^{\widehat{\alpha}}\left(d t_{1}\right)^{\widehat{\alpha}}=\frac{\Gamma(1+\widehat{\alpha})}{\Gamma(1+2 \widehat{\alpha})}(1-\omega)^{2 \widehat{\alpha}}, \\
& \frac{1}{\Gamma(1+\widehat{\alpha})} \int_{1-\omega}^{1}\left(1-t_{1}\right)^{\widehat{\alpha}}\left(d t_{1}\right)^{\widehat{\alpha}}=\frac{\Gamma(1+\widehat{\alpha})}{\Gamma(1+2 \widehat{\alpha})} \omega^{2 \widehat{\alpha}} .
\end{aligned}
$$

Setting $t_{1}=(1-\omega) \eta$, with the aid of Lemma 7 and simple computations yield

$$
\begin{aligned}
\Lambda_{1}^{\widehat{\alpha}}\left(q, \omega, a_{1}, a_{2} ; s\right) \\
:=\frac{1}{\Gamma(1+\widehat{\alpha})} \int_{0}^{1-\omega} t_{1}^{(s+1) \widehat{\alpha}}\left(\frac{a_{1}\left(a_{1}+\mathscr{F}_{\rho, \sigma}^{\lambda}\left(a_{2}-a_{1}\right)\right)}{a_{1}+t_{1} \mathscr{F}_{\rho, \sigma}^{\lambda}\left(a_{2}-a_{1}\right)}\right)^{2 q \widehat{\alpha}}\left(d t_{1}\right)^{\widehat{\alpha}} \\
=\frac{\left(a_{1}\left(a_{1}+\mathscr{F}_{\rho, \sigma}^{\lambda}\left(a_{2}-a_{1}\right)\right)\right)^{2 q \widehat{\alpha}}}{\Gamma(1+\widehat{\alpha})} \int_{0}^{1-\omega} t_{1}^{(s+1) \widehat{\alpha}} \\
\quad \times\left(a_{1}+t_{1} \mathscr{F}_{\rho, \sigma}^{\lambda}\left(a_{2}-a_{1}\right)\right)^{-2 q \widehat{\alpha}}\left(d t_{1}\right)^{\widehat{\alpha}}
\end{aligned}
$$




$$
\begin{aligned}
= & (1-\omega)^{(s+2) \widehat{\alpha}} \frac{\left(a_{1}+\mathscr{F}_{\rho, \sigma}^{\lambda}\left(a_{2}-a_{1}\right)\right)^{2 q \widehat{\alpha}}}{\Gamma(1+\widehat{\alpha})} \int_{0}^{1} t_{1}^{(s+1) \widehat{\alpha}} \\
& \times\left(1-\eta \frac{(\omega-1) \mathscr{F}_{\rho, \sigma}^{\lambda}\left(a_{2}-a_{1}\right)}{a_{1}}\right)^{-2 q \widehat{\alpha}}(d \eta)^{\widehat{\alpha}} \\
= & (1-\omega)^{(s+2) \widehat{\alpha}}\left(a_{1}+\mathscr{F}_{\rho, \sigma}^{\lambda}\left(a_{2}-a_{1}\right)\right)^{2 \widehat{q \alpha} \Gamma(1+(s+1) \widehat{\alpha})} \frac{\widehat{\Gamma}(1+(s+2) \widehat{\alpha})_{2}}{\Gamma(1)} \\
& \times\left(2 q, s+2 ; s+3 ; \frac{(\omega-1) \mathscr{F}_{\rho, \sigma}^{\lambda}\left(a_{2}-a_{1}\right)}{a_{1}}\right) .
\end{aligned}
$$

In a similar way, we can find

$$
\begin{aligned}
& \Lambda_{2}^{\widehat{\alpha}}\left(q, \omega, a_{1}, a_{2} ; s\right) \\
& :=\frac{1}{\Gamma(1+\widehat{\alpha})} \int_{0}^{1-\omega} t_{1}^{\widehat{\alpha}}\left(1-t_{1}\right)^{s \widehat{\alpha}}\left(\frac{a_{1}\left(a_{1}+\mathscr{F}_{\rho, \sigma}^{\lambda}\left(a_{2}-a_{1}\right)\right)}{a_{1}+t_{1} \mathscr{F}_{\rho, \sigma}^{\lambda}\left(a_{2}-a_{1}\right)}\right)^{2 \hat{\alpha} \widehat{\alpha}}\left(d t_{1}\right)^{\widehat{\alpha}} \\
& =\frac{\left(a_{1}\left(a_{1}+\mathscr{F}_{\rho, \sigma}^{\lambda}\left(a_{2}-a_{1}\right)\right)\right)^{2 q \widehat{\alpha}}}{\Gamma(1+\widehat{\alpha})} \int_{0}^{1-\omega}\left[t_{1}^{\widehat{\alpha}}\left(a_{1}+t_{1} \mathscr{F}_{\rho, \sigma}^{\lambda}\left(a_{2}-a_{1}\right)\right)^{-2 q \widehat{\alpha}}\right. \\
& \left.-t_{1}^{(s+1) \widehat{\alpha}}\left(a_{1}+t_{1} \mathscr{F}_{\rho, \sigma}^{\lambda}\left(a_{2}-a_{1}\right)\right)^{-2 q \widehat{\alpha}}\right]\left(d t_{1}\right)^{\widehat{\alpha}} \\
& =(1-\omega)^{2 \widehat{\alpha}}\left(a_{1}+\mathscr{F}_{\rho, \sigma}^{\lambda}\left(a_{2}-a_{1}\right)\right)^{2 q \widehat{\alpha}} \frac{\Gamma(1+\widehat{\alpha})}{\Gamma(1+2 \widehat{\alpha})_{2}} \bar{F}_{1}^{\widehat{\alpha}} \\
& \times\left(2 q, 2 ; 3 ; \frac{(\omega-1) \mathscr{F}_{\rho, \sigma}^{\lambda}\left(a_{2}-a_{1}\right)}{a_{1}}\right)-(1-\omega)^{(s+2) \widehat{\alpha}} \\
& \times\left(a_{1}+\mathscr{F}_{\rho, \sigma}^{\lambda}\left(a_{2}-a_{1}\right)\right)^{2 q \widehat{\alpha}} \frac{\Gamma(1+(s+1) \widehat{\alpha})}{\Gamma(1+(s+2) \widehat{\alpha})_{2}} \widehat{F}_{1}^{\widehat{\alpha}} \\
& \times\left(2 q, s+2 ; s+3 ; 1-\frac{(\omega-1) \mathscr{F}_{\rho, \sigma}^{\lambda}\left(a_{2}-a_{1}\right)}{a_{1}}\right) \\
& =(1-\omega)^{2 \widehat{\alpha}}\left(a_{1}+\mathscr{F}_{\rho, \sigma}^{\lambda}\left(a_{2}-a_{1}\right)\right)^{2 q \widehat{\alpha}} \frac{\Gamma(1+\widehat{\alpha})}{\Gamma(1+2 \widehat{\alpha})_{2}} \bar{F}_{1}^{\widehat{\alpha}} \\
& \times\left(2 q, 2 ; 3 ; \frac{(\omega-1) \mathscr{F}_{\rho, \sigma}^{\lambda}\left(a_{2}-a_{1}\right)}{a_{1}}\right)-\Lambda_{1}^{\widehat{\alpha}}\left(q, \omega, a_{1}, a_{2} ; s\right),
\end{aligned}
$$

$$
\begin{aligned}
\Lambda_{3}^{\widehat{\alpha}}\left(q, \omega, a_{1}, a_{2} ; s\right) & =\frac{1}{\Gamma(1+\widehat{\alpha})} \int_{1-\omega}^{1} t_{1}^{s \hat{\alpha}}\left(1-t_{1}\right)^{\widehat{\alpha}}\left(\frac{a_{1}\left(a_{1}+\mathscr{F}_{\rho, \sigma}^{\lambda}\left(a_{2}-a_{1}\right)\right)}{a_{1}+t_{1} \mathscr{F}_{\rho, \sigma}^{\lambda}\left(a_{2}-a_{1}\right)}\right)^{2 q \widehat{\alpha}}\left(d t_{1}\right)^{\widehat{\alpha}} \\
= & \frac{\left(a_{1}\left(a_{1}+\mathscr{F}_{\rho, \sigma}^{\lambda}\left(a_{2}-a_{1}\right)\right)\right)^{2 q \widehat{\alpha}}}{\Gamma(1+\widehat{\alpha})} \int_{0}^{1}\left[t_{1}^{s \hat{\alpha}}\left(a_{1}+t_{1} \mathscr{F}_{\rho, \sigma}^{\lambda}\left(a_{2}-a_{1}\right)\right)^{-2 q \widehat{\alpha}}\right. \\
& \left.-t_{1}^{(s+1) \widehat{\alpha}}\left(a_{1}+t_{1} \mathscr{F}_{\rho, \sigma}^{\lambda}\left(a_{2}-a_{1}\right)\right)^{-2 q \widehat{\alpha}}\right]\left(d t_{1}\right)^{\widehat{\alpha}} \\
& -\frac{\left(a_{1}\left(a_{1}+\mathscr{F}_{\rho, \sigma}^{\lambda}\left(a_{2}-a_{1}\right)\right)\right)^{2 q \widehat{\alpha}}}{\Gamma(1+\widehat{\alpha})} \int_{0}^{1-\omega}
\end{aligned}
$$

$$
\begin{aligned}
& \times\left[t_{1}^{s \widehat{\alpha}}\left(a_{1}+t_{1} \mathscr{F}_{\rho, \sigma}^{\lambda}\left(a_{2}-a_{1}\right)\right)^{-2 q \widehat{\alpha}}-t_{1}^{(s+1) \widehat{\alpha}}\right. \\
& \left.\times\left(a_{1}+t_{1} \mathscr{F}_{\rho, \sigma}^{\lambda}\left(a_{2}-a_{1}\right)\right)^{-2 q \widehat{\alpha}}\right]\left(d t_{1}\right)^{\widehat{\alpha}} \\
= & \left(a_{1}+\mathscr{F}_{\rho, \sigma}^{\lambda}\left(a_{2}-a_{1}\right)\right)^{2 q \widehat{\alpha}}\left[\frac{\Gamma(1+\widehat{\alpha})}{\Gamma(1+(s+1) \widehat{\alpha})_{2}} \bar{F}_{1}^{\widehat{\alpha}}\right. \\
& \times\left(2 q, s+1 ; s+2 ; \frac{(\omega-1) \mathscr{F}_{\rho, \sigma}^{\lambda}\left(a_{2}-a_{1}\right)}{a_{1}}\right) \\
& -\frac{\Gamma(1+(s+2) \widehat{\alpha})}{\Gamma(1+(s+3) \widehat{\alpha})_{2}} \bar{F}_{1}^{\widehat{\alpha}}\left(2 q, s+2 ; s+3 ; \frac{(\omega-1) \mathscr{F}_{\rho, \sigma}^{\lambda}\left(a_{2}-a_{1}\right)}{a_{1}}\right) \\
& -(1-\omega)^{(s+1) \widehat{\alpha}} \frac{\Gamma(1+s \widehat{\alpha})}{\Gamma(1+(s+1) \widehat{\alpha})} \times{ }_{2} \bar{F}_{1}^{\alpha} \\
& \left.\times\left(2 q,(s+1) ;(s+2) ; \frac{(\omega-1) \mathscr{F}_{\rho, \sigma}^{\lambda}\left(a_{2}-a_{1}\right)}{a_{1}}\right)\right] \\
& +\Lambda_{1}^{\widehat{\alpha}}\left(q, \omega, a_{1}, a_{2} ; s\right),
\end{aligned}
$$

$\Lambda_{4}^{\widehat{\alpha}}\left(q, \omega, a_{1}, a_{2} ; s\right)$

$$
\begin{aligned}
:= & \frac{1}{\Gamma(1+\widehat{\alpha})} \int_{1-\omega}^{1}\left(1-t_{1}\right)^{(s+1) \widehat{\alpha}}\left(\frac{a_{1}\left(a_{1}+\mathscr{F}_{\rho, \sigma}^{\lambda}\left(a_{2}-a_{1}\right)\right)}{a_{1}+t_{1} \mathscr{F}_{\rho, \sigma}^{\lambda}\left(a_{2}-a_{1}\right)}\right)^{2 q \widehat{\alpha}}\left(d t_{1}\right)^{\widehat{\alpha}} \\
= & \frac{\left(a_{1}\left(a_{1}+\mathscr{F}_{\rho, \sigma}^{\lambda}\left(a_{2}-a_{1}\right)\right)\right)^{2 q \widehat{\alpha}}}{\Gamma(1+\widehat{\alpha})} \int_{0}^{1}\left(1-t_{1}\right)^{(s+1) \widehat{\alpha}} \\
& \times\left(a_{1}+t_{1} \mathscr{F}_{\rho, \sigma}^{\lambda}\left(a_{2}-a_{1}\right)\right)^{-2 q \widehat{\alpha}}\left(d t_{1}\right)^{\widehat{\alpha}} \\
& -\frac{\left(a_{1}\left(a_{1}+\mathscr{F}_{\rho, \sigma}^{\lambda}\left(a_{2}-a_{1}\right)\right)\right)^{2 q \widehat{\alpha}}}{\Gamma(1+\widehat{\alpha})} \int_{0}^{1-\omega}\left(1-t_{1}\right)^{(s+1) \widehat{\alpha}} \\
& \times\left(a_{1}+t_{1} \mathscr{F}_{\rho, \sigma}^{\lambda}\left(a_{2}-a_{1}\right)\right)^{-2 q \widehat{\alpha}}\left(d t_{1}\right)^{\widehat{\alpha}} \\
= & \left(a_{1}+\mathscr{F}_{\rho, \sigma}^{\lambda}\left(a_{2}-a_{1}\right)\right)^{2 q \widehat{\alpha}}\left[\frac{\Gamma(1+(s+2) \widehat{\alpha})}{\Gamma(1+(s+3) \widehat{\alpha})_{2}} \bar{F}_{1}^{\widehat{\alpha}}\right. \\
& \times\left(2 q, 1 ; s+3 ; \frac{(\omega-1) \mathscr{F}_{\rho, \sigma}^{\lambda}\left(a_{2}-a_{1}\right)}{a_{1}}\right)-(1-\omega)^{\widehat{\alpha}} \Gamma(1+\widehat{\alpha})_{2} \bar{F}_{1}^{\widehat{\alpha}} \\
& \times\left(2 q, 1 ; 2 ; \frac{(\omega-1) \mathscr{F}_{\rho, \sigma}^{\lambda}\left(a_{2}-a_{1}\right)}{a_{1}}\right)+(1-\omega)^{(s+2) \widehat{\alpha}} \\
& \left.\times \frac{\Gamma(1+(s+2) \widehat{\alpha})}{\Gamma(1+(s+3) \widehat{\alpha})_{2}} \widehat{F}_{1}^{\widehat{\alpha}}\left(2 q, s+2 ; s+3 ; \frac{(\omega-1) \mathscr{F}_{\rho, \sigma}^{\lambda}\left(a_{2}-a_{1}\right)}{a_{1}}\right)\right] .
\end{aligned}
$$

A combination of (41)-(44) with (39), we get the desired inequality (37). This gives the proof of Theorem 17.

From Theorem 17, we get the following corollaries immediately. 
Corollary 18. Let $\omega=0$. Then, Theorem 17 leads to the following local fractional integral inequality of "Ostrowski type"

$$
\begin{aligned}
\mid Q\left(a_{2}\right) & -\left(\frac{\mathscr{F}_{\rho, \sigma}^{\lambda}\left(a_{2}-a_{1}\right)}{a_{1}\left(a_{1}+\mathscr{F}_{\rho, \sigma}^{\lambda}\left(a_{2}-a_{1}\right)\right)}\right)^{\widehat{\alpha}} \Gamma(1+\widehat{\alpha})_{a_{1}} \mathscr{F}_{a_{1}+\mathscr{F}_{\rho, \sigma}^{\lambda}\left(a_{2}-a_{1}\right)}^{(\widehat{Q})} \frac{Q(z)}{z^{2 \widehat{\alpha}}} \mid \\
\leq & \frac{\left(\mathscr{F}_{\rho, \sigma}^{\lambda}\left(a_{2}-a_{1}\right)\right)^{\widehat{\alpha}}}{a_{1}^{\widehat{\alpha}}\left(a_{1}+\mathscr{F}_{\rho, \sigma}^{\lambda}\left(a_{2}-a_{1}\right)\right)^{\widehat{\alpha}}}\left(\frac{\Gamma(1+\widehat{\alpha})}{\Gamma(1+2 \widehat{\alpha})}\right)^{1-1 / q} \\
& \times\left\{\left[\Lambda_{1}^{\widehat{\alpha}}\left(q, 0, a_{1}, a_{2} ; s\right)\left|Q^{(\widehat{\alpha})}\left(a_{1}\right)\right|^{q}+\Lambda_{2}^{\widehat{\alpha}}\left(q, 0, a_{1}, a_{2} ; s\right)\right.\right. \\
& \left.\left.\times\left|Q^{(\widehat{\alpha})}\left(a_{2}\right)\right|^{q}\right]\right\}^{1 / q},
\end{aligned}
$$

where

$$
\begin{aligned}
& \Lambda_{1}^{\widehat{\alpha}}\left(q, 0, a_{1}, a_{2} ; s\right) \\
& :=\left(a_{1}+\mathscr{F}_{\rho, \sigma}^{\lambda}\left(a_{2}-a_{1}\right)\right)^{2 q \widehat{\alpha}} \times \frac{\Gamma(1+(s+1) \widehat{\alpha})}{\Gamma(1+(s+2) \widehat{\alpha})_{2}} \bar{F}_{1}^{\widehat{\alpha}} \\
& \left(2 q, s+2 ; s+3 ; \frac{-\mathscr{F}_{\rho, \sigma}^{\lambda}\left(a_{2}-a_{1}\right)}{a_{1}}\right), \\
& \Lambda_{2}^{\widehat{\alpha}}\left(q, 0, a_{1}, a_{2} ; s\right) \\
& :=\left(a_{1}+\mathscr{F}_{\rho, \sigma}^{\lambda}\left(a_{2}-a_{1}\right)\right)^{2 q \widehat{\alpha}} \frac{\Gamma(1+\widehat{\alpha})}{\Gamma(1+2 \widehat{\alpha})} \\
& \times{ }_{2} \bar{F}_{1}^{\widehat{\alpha}}\left(2 q, 2 ; 3 ; \frac{-\mathscr{F}_{\rho, \sigma}^{\lambda}\left(a_{2}-a_{1}\right)}{a_{1}}\right) \\
& -\Lambda_{1}^{\widehat{\alpha}}\left(q, 0, a_{1}, a_{2} ; s\right) \text {. } \\
& \left|Q\left(a_{1}\right)-\left(\frac{\mathscr{F}_{\rho, \sigma}^{\lambda}\left(a_{2}-a_{1}\right)}{a_{1}\left(a_{1}+\mathscr{F}_{\rho, \sigma}^{\lambda}\left(a_{2}-a_{1}\right)\right)}\right)^{\widehat{\alpha}} \Gamma(1+\widehat{\alpha})_{a_{1}} \mathscr{F}_{a_{1}+\mathscr{F}_{\rho, \sigma}^{\lambda}\left(a_{2}-a_{1}\right)}^{(\widehat{(})} \frac{Q(z)}{z^{2 \widehat{\alpha}}}\right| \\
& \leq \frac{\left(\mathscr{F}_{\rho, \sigma}^{\lambda}\left(a_{2}-a_{1}\right)\right)^{\widehat{\alpha}}}{a_{1}^{\widehat{\alpha}}\left(a_{1}+\mathscr{F}_{\rho, \sigma}^{\lambda}\left(a_{2}-a_{1}\right)\right)^{\widehat{\alpha}}}\left(\frac{\Gamma(1+\widehat{\alpha})}{\Gamma(1+2 \widehat{\alpha})}\right)^{1-1 / q} \\
& \times\left\{\left[\Lambda_{3}^{\widehat{\alpha}}\left(q, 1, a_{1}, a_{2} ; s\right)\left|Q^{(\widehat{\alpha})}\left(a_{1}\right)\right|^{q}\right.\right. \\
& \left.\left.+\Lambda_{4}^{\widehat{\alpha}}\left(q, 1, a_{1}, a_{2} ; s\right)\left|Q^{(\widehat{\alpha})}\left(a_{2}\right)\right|^{q}\right]\right\}^{1 / q},
\end{aligned}
$$

where

$$
\begin{aligned}
\Lambda_{3}^{\widehat{\alpha}}\left(q, 1, a_{1}, a_{2} ; s\right) \\
:=\left(a_{1}+\mathscr{F}_{\rho, \sigma}^{\lambda}\left(a_{2}-a_{1}\right)\right)^{2 q \widehat{\alpha}}\left[\frac{\Gamma(1+(s+1) \widehat{\alpha})}{\Gamma(1+(s+2) \widehat{\alpha})_{2}} \widehat{F}_{1}^{\widehat{\alpha}}\right. \\
\quad \times\left(2 q, s+1 ; s+2 ; \frac{-\mathscr{F}_{\rho, \sigma}^{\lambda}\left(a_{2}-a_{1}\right)}{a_{1}}\right) \\
\left.\quad-\frac{\Gamma(1+(s+1) \widehat{\alpha})}{\Gamma(1+(s+2) \widehat{\alpha})_{2}} \bar{F}_{1}^{\widehat{\alpha}}\left(2 q, s+2 ; s+3 ; \frac{-\mathscr{F}_{\rho, \sigma}^{\lambda}\left(a_{2}-a_{1}\right)}{a_{1}}\right)\right], \\
\Lambda_{4}^{\widehat{\alpha}}\left(q, 1, a_{1}, a_{2} ; s\right) \\
:=\left(a_{1}+\mathscr{F}_{\rho, \sigma}^{\lambda}\left(a_{2}-a_{1}\right)\right)^{2 q \widehat{\alpha}} \frac{\Gamma(1+(s+2) \widehat{\alpha})}{\Gamma(1+(s+3) \widehat{\alpha})_{2}} \bar{F}_{1}^{\widehat{\alpha}} \\
\quad \times\left(2 q, 1 ; s+3 ; \frac{-\mathscr{F}_{\rho, \sigma}^{\lambda}\left(a_{2}-a_{1}\right)}{a_{1}}\right) .
\end{aligned}
$$

Corollary 20. By means of Corollary 18 and Corollary 19. Then, Theorem 17 leads to the following local fractional integral inequality of "trapezoid type"

$$
\begin{aligned}
& \mid \frac{Q\left(a_{1}\right)+Q\left(a_{2}\right)}{2^{\widehat{\alpha}}}-\left(\frac{\mathscr{F}_{\rho, \sigma}^{\lambda}\left(a_{2}-a_{1}\right)}{a_{1}\left(a_{1}+\mathscr{F}_{\rho, \sigma}^{\lambda}\left(a_{2}-a_{1}\right)\right)}\right)^{\widehat{\alpha}} \Gamma \\
& \times(1+\widehat{\alpha})_{a_{1}} \mathscr{I} \underset{a_{1}+\mathscr{F}_{\rho, \sigma}^{\lambda}\left(a_{2}-a_{1}\right)}{(\widehat{Q})} \frac{Q(z)}{z^{2 \widehat{\alpha}}} \mid \\
& \leq \frac{\left(\mathscr{F}_{\rho, \sigma}^{\lambda}\left(a_{2}-a_{1}\right)\right)^{\widehat{\alpha}}}{2^{\widehat{\alpha}} a_{1}^{\widehat{\alpha}}\left(a_{1}+\mathscr{F}_{\rho, \sigma}^{\lambda}\left(a_{2}-a_{1}\right)\right)^{\widehat{\alpha}}}\left(\frac{\Gamma(1+\widehat{\alpha})}{\Gamma(1+2 \widehat{\alpha})}\right)^{1-1 / q} \\
& \times\left[\left\{\left[\Lambda_{1}^{\widehat{\alpha}}\left(q, 0, a_{1}, a_{2} ; s\right)\left|Q^{(\widehat{\alpha})}\left(a_{1}\right)\right|^{q}+\Lambda_{2}^{\widehat{\alpha}}\left(q, 0, a_{1}, a_{2} ; s\right)\right.\right.\right. \\
& \left.\left.\times\left|Q^{(\widehat{\alpha})}\left(a_{2}\right)\right|^{q}\right]\right\}^{1 / q}+\left\{\left[\Lambda_{3}^{\widehat{\alpha}}\left(q, 1, a_{1}, a_{2} ; s\right)\left|Q^{(\widehat{\alpha})}\left(a_{1}\right)\right|^{q}\right.\right. \\
& \left.\left.\left.+\Lambda_{4}^{\widehat{\alpha}}\left(q, 1, a_{1}, a_{2} ; s\right)\left|Q^{(\widehat{\alpha})}\left(a_{2}\right)\right|^{q}\right]\right\}^{1 / q}\right],
\end{aligned}
$$

where $\Lambda_{1}^{\widehat{\alpha}}\left(q, 0, a_{1}, a_{2} ; s\right), \Lambda_{2}^{\widehat{\alpha}}\left(q, 0, a_{1}, a_{2} ; s\right), \Lambda_{3}^{\widehat{\alpha}}\left(q, 1, a_{1}, a_{2}\right.$; $s)$, and $\Lambda_{4}^{\alpha}\left(q, 1, a_{1}, a_{2} ; s\right)$ are given in Corollary 18 and Corollary 19, respectively.

Corollary 21. Let $\omega=1 / 2$. Then, Theorem 17 leads to the following local fractional integral inequality of "midpoint type"

$$
\begin{gathered}
\mid Q\left(\frac{2 a_{1} a_{2}}{a_{1}+a_{2}}\right)-\left(\frac{\mathscr{F}_{\rho, \sigma}^{\lambda}\left(a_{2}-a_{1}\right)}{a_{1}\left(a_{1}+\mathscr{F}_{\rho, \sigma}^{\lambda}\left(a_{2}-a_{1}\right)\right)}\right)^{\widehat{\alpha}} \Gamma \\
\times(1+\widehat{\alpha})_{a_{1}} \mathcal{I}_{a_{1}+\mathscr{F}_{\rho, \sigma}^{\lambda}\left(a_{2}-a_{1}\right)}^{(\widehat{Q})} \frac{Q(z)}{z^{2 \widehat{\alpha}}} \mid
\end{gathered}
$$


Journal of Function Spaces

11

$$
\begin{aligned}
& \left.\leq \frac{\left(\mathscr{F}_{\rho, \sigma}^{\lambda}\left(a_{2}-a_{1}\right)\right)^{\widehat{\alpha}}}{a_{1}^{\widehat{\alpha}}\left(a_{1}+\mathscr{F}_{\rho, \sigma}^{\lambda}\left(a_{2}-a_{1}\right)\right)^{\widehat{\alpha}}\left(\frac{\Gamma(1+\widehat{\alpha})}{4^{\alpha}} \Gamma(1+2 \widehat{\alpha})\right.}\right)^{1-1 / q} \\
& \quad \times\left[\left\{\left[\Lambda_{1}^{\widehat{\alpha}}\left(q, \frac{1}{2}, a_{1}, a_{2} ; s\right)\left|Q^{(\widehat{\alpha})}\left(a_{1}\right)\right|^{q}+\Lambda_{2}^{\widehat{\alpha}}\right.\right.\right. \\
& \left.\left.\quad \times\left(q, \frac{1}{2}, a_{1}, a_{2} ; s\right)\left|Q^{(\widehat{\alpha})}\left(a_{2}\right)\right|^{q}\right]\right\}^{1 / q} \\
& +\left\{\left[\Lambda_{3}^{\widehat{\alpha}}\left(q, \frac{1}{2}, a_{1}, a_{2} ; s\right)\left|Q^{(\widehat{\alpha})}\left(a_{1}\right)\right|^{q}+\Lambda_{4}^{\widehat{\alpha}}\right.\right. \\
& \left.\left.\left.\quad \times\left(q, \frac{1}{2}, a_{1}, a_{2} ; s\right)\left|Q^{(\widehat{\alpha})}\left(a_{2}\right)\right|^{q}\right]\right\}^{1 / q}\right],
\end{aligned}
$$

where

$$
\begin{aligned}
& \Lambda_{1}^{\widehat{\alpha}}\left(q, \frac{1}{2}, a_{1}, a_{2} ; s\right) \\
& :=\left(\frac{1}{2}\right)^{(s+2) \widehat{\alpha}}\left(a_{1}+\mathscr{F}_{\rho, \sigma}^{\lambda}\left(a_{2}-a_{1}\right)\right)^{2 q \widehat{\alpha}} \times \frac{\Gamma(1+(s+1) \widehat{\alpha})}{\Gamma(1+(s+2) \widehat{\alpha})_{2}} \bar{F}_{1}^{\widehat{\alpha}} \\
& \times\left(2 q, s+2 ; s+3 ; \frac{-\mathscr{F}_{\rho, \sigma}^{\lambda}\left(a_{2}-a_{1}\right)}{2 a_{1}}\right), \\
& \Lambda_{2}^{\widehat{\alpha}}\left(q, \frac{1}{2}, a_{1}, a_{2} ; s\right) \\
& :=\left(\frac{1}{2}\right)^{2 \widehat{\alpha}}\left(a_{1}+\mathscr{F}_{\rho, \sigma}^{\lambda}\left(a_{2}-a_{1}\right)\right)^{2 q \widehat{\alpha}} \frac{\Gamma(1+\widehat{\alpha})}{\Gamma(1+2 \widehat{\alpha})} \times{ }_{2} \bar{F}_{1}^{\widehat{\alpha}} \\
& \times\left(2 q, 2 ; 3 ; \frac{\mathscr{F}_{\rho, \sigma}^{\lambda}\left(a_{2}-a_{1}\right)}{2 a_{1}}\right)-\Lambda_{1}^{\hat{\alpha}}\left(q, \frac{1}{2}, a_{1}, a_{2} ; s\right), \\
& \Lambda_{3}^{\widehat{\alpha}}\left(q, \frac{1}{2}, a_{1}, a_{2} ; s\right) \\
& :=\left(a_{1}+\mathscr{F}_{\rho, \sigma}^{\lambda}\left(a_{2}-a_{1}\right)\right)^{2 q \widehat{\alpha}}\left[\frac{\Gamma(1+s \widehat{\alpha})}{\Gamma(1+(s+1) \widehat{\alpha})_{2}} \bar{F}_{1}^{\widehat{\alpha}}\right. \\
& \times\left(2 q, s+1 ; s+2 ; \frac{-\mathscr{F}_{\rho, \sigma}^{\lambda}\left(a_{2}-a_{1}\right)}{2 a_{1}}\right) \\
& -\frac{\Gamma(1+(s+2) \widehat{\alpha})}{\Gamma(1+(s+3) \widehat{\alpha})_{2}} \bar{F}_{1}^{\widehat{\alpha}}\left(2 q, s+2 ; s+3 ; \frac{-\mathscr{F}_{\rho, \sigma}^{\lambda}\left(a_{2}-a_{1}\right)}{2 a_{1}}\right) \\
& -\left(\frac{1}{2}\right)^{(s+1) \widehat{\alpha}} \frac{\Gamma(1+s \widehat{\alpha})}{\Gamma(1+(s+1) \widehat{\alpha})} \times{ }_{2} \bar{F}_{1}^{\widehat{\alpha}} \\
& \left.\times\left(2 q,(s+1) ;(s+2) ; \frac{-\mathscr{F}_{\rho, \sigma}^{\lambda}\left(a_{2}-a_{1}\right)}{2 a_{1}}\right)\right] \\
& +\Lambda_{1}^{\widehat{\alpha}}\left(q, \frac{1}{2}, a_{1}, a_{2} ; s\right),
\end{aligned}
$$

$$
\begin{aligned}
\Lambda_{4}^{\widehat{\alpha}}\left(q, \frac{1}{2}, a_{1}, a_{2} ; s\right) \\
:=\left(a_{1}+\mathscr{F}_{\rho, \sigma}^{\lambda}\left(a_{2}-a_{1}\right)\right)^{2 q \widehat{\alpha}}\left[\frac{\Gamma(1+(s+2) \widehat{\alpha})}{\Gamma(1+(s+3) \widehat{\alpha})_{2}} \bar{F}_{1}^{\widehat{\alpha}}\right. \\
\quad \times\left(2 q, 1 ; s+3 ; \frac{-\mathscr{F}_{\rho, \sigma}^{\lambda}\left(a_{2}-a_{1}\right)}{2 a_{1}}\right)-\left(\frac{1}{2}\right)^{\widehat{\alpha}} \Gamma(1+\widehat{\alpha})_{2} \bar{F}_{1}^{\widehat{\alpha}} \\
\quad \times\left(2 q, 1 ; 2 ; \frac{-\mathscr{F}_{\rho, \sigma}^{\lambda}\left(a_{2}-a_{1}\right)}{2 a_{1}}\right)+(1-\omega)^{(s+2) \widehat{\alpha}} \\
\left.\quad \times \frac{\Gamma(1+(s+2) \widehat{\alpha})}{\Gamma(1+(s+3) \widehat{\alpha})_{2}} \bar{F}_{1}^{\alpha}\left(2 q, s+2 ; s+3 ; \frac{-\mathscr{F}_{\rho, \sigma}^{\lambda}\left(a_{2}-a_{1}\right)}{2 a_{1}}\right)\right] .
\end{aligned}
$$

Theorem 22. Assume that the hypothesis of Lemma 16 are satisfied. If $\left|Q^{(\widehat{\alpha})}\right|^{q}$ is a generalized harmonically $\psi$ - s-convex function on $\mathscr{I}_{\mathscr{F}}$ for $p, q>1, p^{-1}+q^{-1}=1$. Then, the following inequality for local fractional integrals holds

$$
\begin{aligned}
& \left|\Omega_{Q}\left(a_{1}, a_{1}+\mathscr{F}_{\rho, \sigma}^{\lambda}\left(a_{2}-a_{1}\right) ; \omega, \widehat{\alpha}\right)\right| \\
& \leq \frac{\left(\mathscr{F}_{\rho, \sigma}^{\lambda}\left(a_{2}-a_{1}\right)\right)^{\widehat{\alpha}}}{a_{1}^{\widehat{\alpha}}\left(a_{1}+\mathscr{F}_{\rho, \sigma}^{\lambda}\left(a_{2}-a_{1}\right)\right)^{\widehat{\alpha}}}\left(\frac{\Gamma(1+s \widehat{\alpha})}{\Gamma(1+(s+1) \widehat{\alpha})}\right)^{1 / q} \\
& \times\left\{( \Lambda _ { 5 } ^ { \widehat { \alpha } } ( p , \omega , a _ { 1 } , a _ { 2 } ; s ) ) ^ { 1 / p } \left[(1-\omega)^{(s+1) \widehat{\alpha}}\left|Q^{(\widehat{\alpha})}\left(a_{1}\right)\right|^{q}\right.\right. \\
& \left.+\left[1^{\widehat{\alpha}}-\omega^{(s+1) \widehat{\alpha}}\right]\left|Q^{(\widehat{\alpha})}\left(a_{2}\right)\right|^{q}\right]^{1 / q}+\left(\Lambda_{6}^{\widehat{\alpha}}\left(p, \omega, a_{1}, a_{2} ; s\right)\right)^{1 / p} \\
& \left.\times\left[\left[1^{\widehat{\alpha}}-(1-\omega)^{(s+1) \widehat{\alpha}}\right]\left|Q^{(\widehat{\alpha})}\left(a_{1}\right)\right|^{q}+\omega^{(s+1) \widehat{\alpha}}\left|Q^{(\widehat{\alpha})}\left(a_{2}\right)\right|^{q}\right]^{1 / q}\right\},
\end{aligned}
$$

where

$$
\begin{aligned}
& \Lambda_{5}^{\widehat{\alpha}}\left(p, \omega, a_{1}, a_{2} ; s\right) \\
& :=\left(a_{1}+\mathscr{F}_{\rho, \sigma}^{\lambda}\left(a_{2}-a_{1}\right)\right)^{2 \hat{p} \widehat{\alpha}}(1-\omega)^{(p+1) \widehat{\alpha}} \frac{\Gamma(1+p \widehat{\alpha})}{\Gamma(1+(p+1) \widehat{\alpha})} \\
& \times{ }_{2} \bar{F}_{1}^{\widehat{\alpha}}\left(2 p, p+1 ; p+2 ; \frac{(\omega-1) \mathscr{F}_{\rho, \sigma}^{\lambda}\left(a_{2}-a_{1}\right)}{a_{1}}\right), \\
& \Lambda_{6}^{\widehat{\alpha}}\left(p, \omega, a_{1}, a_{2} ; s\right) \\
& :=\left(a_{1}+\mathscr{F}_{\rho, \sigma}^{\lambda}\left(a_{2}-a_{1}\right)\right)^{2 p \widehat{\alpha}}\left[\frac{\Gamma(1+(p+2) \widehat{\alpha})}{\Gamma(1+(p+3) \widehat{\alpha})_{2}} \bar{F}_{1}^{\widehat{\alpha}}\right. \\
& \times\left(2 p, 1 ; p+2 ; \frac{-\mathscr{F}_{\rho, \sigma}^{\lambda}\left(a_{2}-a_{1}\right)}{a_{1}}\right)-\Gamma(1+\widehat{\alpha})_{2} \bar{F}_{1}^{\alpha} \\
& \times\left(2 p, 1 ; 2 ; \frac{-\mathscr{F}_{\rho, \sigma}^{\lambda}\left(a_{2}-a_{1}\right)}{a_{1}}\right)+(1-\omega)^{\hat{p \alpha}} \frac{\Gamma(1+(p+1) \widehat{\alpha})}{\Gamma(1+(p+2) \widehat{\alpha})} \\
& \left.\times{ }_{2} \bar{F}_{1}^{\alpha}\left(2 p, p+1 ; p+2 ; \frac{-\mathscr{F}_{\rho, \sigma}^{\lambda}\left(a_{2}-a_{1}\right)}{a_{1}}\right)\right] \text {. }
\end{aligned}
$$


Proof. By means of Lemma 16 and utilizing the generalized Hölder inequality, we have

$$
\begin{aligned}
& \left|\Omega_{Q}\left(a_{1}, a_{1}+\mathscr{F}_{\rho, \sigma}^{\lambda}\left(a_{2}-a_{1}\right) ; \omega, \widehat{\alpha}\right)\right| \\
& \leq \frac{\left(\mathscr{F}_{\rho, \sigma}^{\lambda}\left(a_{2}-a_{1}\right)\right)^{\widehat{\alpha}}}{a_{1}^{\widehat{\alpha}}\left(a_{1}+\mathscr{F}_{\rho, \sigma}^{\lambda}\left(a_{2}-a_{1}\right)\right)^{\widehat{\alpha}}}\left[\left(\frac{1}{\Gamma(1+\widehat{\alpha})} \int_{0}^{1-\omega} t_{1}^{p \widehat{\alpha}}\right.\right. \\
& \left.\times\left(\frac{a_{1}\left(a_{1}+\mathscr{F}_{\rho, \sigma}^{\lambda}\left(a_{2}-a_{1}\right)\right)}{a_{1}+t_{1} \mathscr{F}_{\rho, \sigma}^{\lambda}\left(a_{2}-a_{1}\right)}\right)^{2 p \widehat{\alpha}}\left(d t_{1}\right)^{\widehat{\alpha}}\right)^{1 / p} \\
& \times\left(\frac{1}{\Gamma(1+\widehat{\alpha})} \int_{1-\omega}^{1}\left|Q^{(\widehat{\alpha})}\left(\frac{a_{1}\left(a_{1}+\mathscr{F}_{\rho, \sigma}^{\lambda}\left(a_{2}-a_{1}\right)\right)}{a_{1}+t_{1} \mathscr{F}_{\rho, \sigma}^{\lambda}\left(a_{2}-a_{1}\right)}\right)\right|^{q}\left(d t_{1}\right)^{\widehat{\alpha}}\right)^{1 / q} \\
& +\left(\frac{1}{\Gamma(1+\widehat{\alpha})} \int_{1-\omega}^{1}\left(1-t_{1}\right)^{p \hat{\alpha}}\left(\frac{a_{1}\left(a_{1}+\mathscr{F}_{\rho, \sigma}^{\lambda}\left(a_{2}-a_{1}\right)\right)}{a_{1}+t_{1} \mathscr{F}_{\rho, \sigma}^{\lambda}\left(a_{2}-a_{1}\right)}\right)^{2 p \widehat{\alpha}}\right. \\
& \left.\times\left(d t_{1}\right)^{\widehat{\alpha}}\right)^{1 / p} \times\left(\frac{1}{\Gamma(1+\widehat{\alpha})} \int_{1-\omega}^{1} \mid Q^{(\widehat{\alpha})}\right. \\
& \left.\left.\times\left(\frac{a_{1}\left(a_{1}+\mathscr{F}_{\rho, \sigma}^{\lambda}\left(a_{2}-a_{1}\right)\right)}{a_{1}+t_{1} \mathscr{F}_{\rho, \sigma}^{\lambda}\left(a_{2}-a_{1}\right)}\right) \|^{q}\left(d t_{1}\right)^{\hat{\alpha}}\right)^{1 / q}\right] .
\end{aligned}
$$

Since $\left|Q^{(\widehat{\alpha})}\right|^{q}$ is generalized harmonically $\psi$-s-convex function, then we have

$$
\begin{aligned}
& \frac{1}{\Gamma(1+\widehat{\alpha})} \int_{0}^{1-\omega}\left|Q^{(\widehat{\alpha})}\left(\frac{a_{1}\left(a_{1}+\mathscr{F}_{\rho, \sigma}^{\lambda}\left(a_{2}-a_{1}\right)\right)}{a_{1}+t_{1} \mathscr{F}_{\rho, \sigma}^{\lambda}\left(a_{2}-a_{1}\right)}\right)\right|\left(d t_{1}\right)^{\widehat{\alpha}} \\
& \leq \frac{1}{\Gamma(1+\widehat{\alpha})} \int_{0}^{1-\omega}\left[t_{1}^{\widehat{s \alpha}}\left|Q^{(\widehat{\alpha})}\left(a_{1}\right)\right|^{q}+\left(1-t_{1}\right)^{\widehat{s \alpha}}\left|Q^{(\widehat{\alpha})}\left(a_{2}\right)\right|^{q}\right]\left(d t_{1}\right)^{\widehat{\alpha}} \\
& =\frac{\Gamma(1+s \widehat{\alpha})}{\Gamma(1+(s+1) \widehat{\alpha})}\left[(1-\omega)^{(s+1) \hat{\alpha}}\left|Q^{(\widehat{\alpha})}\left(a_{1}\right)\right|^{q}\right. \\
& \left.\quad+\left[1^{\widehat{\alpha}}-\omega^{(s+1) \hat{\alpha}}\right]\left|Q^{(\widehat{\alpha})}\left(a_{2}\right)\right|^{q}\right] .
\end{aligned}
$$

Analogously, we have

$$
\begin{aligned}
& \frac{1}{\Gamma(1+\widehat{\alpha})} \int_{1-\omega}^{1}\left|Q^{(\widehat{\alpha})}\left(\frac{a_{1}\left(a_{1}+\mathscr{F}_{\rho, \sigma}^{\lambda}\left(a_{2}-a_{1}\right)\right)}{a_{1}+t_{1} \mathscr{F}_{\rho, \sigma}^{\lambda}\left(a_{2}-a_{1}\right)}\right)\right|\left(d t_{1}\right)^{\widehat{\alpha}} \\
& \leq \frac{\Gamma(1+s \widehat{\alpha})}{\Gamma(1+(s+1) \widehat{\alpha})}\left[\left[1^{\widehat{\alpha}}-(1-\omega)^{(s+1) \widehat{\alpha}}\right]\left|Q^{(\widehat{\alpha})}\left(a_{1}\right)\right|^{q}\right. \\
& \left.+\omega^{(s+1) \hat{\alpha}}\left|Q^{(\widehat{\alpha})}\left(a_{2}\right)\right|^{q}\right] .
\end{aligned}
$$

Setting $t_{1}=(1-\omega) \eta$, with the aid of Lemma 7 and simple computations yield

$$
\begin{aligned}
\Lambda_{5}^{\widehat{\alpha}}\left(p, \omega, a_{1}, a_{2} ; s\right) & =\frac{1}{\Gamma(1+\widehat{\alpha})} \int_{0}^{1-\omega} t_{1}^{p \widehat{\alpha}}\left(\frac{a_{1}\left(a_{1}+\mathscr{F}_{\rho, \sigma}^{\lambda}\left(a_{2}-a_{1}\right)\right)}{a_{1}+t_{1} \mathscr{F}_{\rho, \sigma}^{\lambda}\left(a_{2}-a_{1}\right)}\right)^{2 p \widehat{\alpha}}\left(d t_{1}\right)^{\widehat{\alpha}} \\
= & \left(a_{1}\left(a_{1}+\mathscr{F}_{\rho, \sigma}^{\lambda}\left(a_{2}-a_{1}\right)\right)\right)^{2 p \widehat{\alpha}} \frac{1}{\Gamma(1+\widehat{\alpha})} \int_{0}^{1-\omega} t_{1}^{p \widehat{\alpha}} \\
& \times\left(a_{1}+t_{1} \mathscr{F}_{\rho, \sigma}^{\lambda}\left(a_{2}-a_{1}\right)\right)^{-2 p \widehat{\alpha}}\left(d t_{1}\right)^{\widehat{\alpha}} \\
= & \left(a_{1}+\mathscr{F}_{\rho, \sigma}^{\lambda}\left(a_{2}-a_{1}\right)\right)^{2 p \widehat{\alpha}}(1-\omega)^{(p+1) \widehat{\alpha}} \frac{1}{\Gamma(1+\widehat{\alpha})} \int_{0}^{1} \eta^{p \widehat{\alpha}} \\
& \times\left(1-\frac{(\omega-1) \mathscr{F}_{\rho, \sigma}^{\lambda}\left(a_{2}-a_{1}\right)}{a_{1}}\right)^{-2 p \widehat{\alpha}}\left(d t_{1}\right)^{\widehat{\alpha}} \\
= & \left(a_{1}+\mathscr{F}_{\rho, \sigma}^{\lambda}\left(a_{2}-a_{1}\right)\right)^{2 p \widehat{\alpha}}(1-\omega)^{(p+1) \widehat{\alpha}} \frac{\Gamma(1+p \widehat{\alpha})}{\Gamma(1+(p+1) \widehat{\alpha})} \\
& \times{ }_{2} \bar{F}_{1}^{\widehat{\alpha}}\left(2 p, p+1 ; p+2 ; \frac{(\omega-1) \mathscr{F}_{\rho, \sigma}^{\lambda}\left(a_{2}-a_{1}\right)}{a_{1}}\right) .
\end{aligned}
$$

In a similar way, we have

$$
\begin{aligned}
\Lambda_{6}^{\widehat{\alpha}}\left(p, \omega, a_{1}, a_{2} ; s\right) & =\frac{1}{\Gamma(1+\widehat{\alpha})} \int_{1-\omega}^{1}\left(1-t_{1}\right)^{p \widehat{\alpha}}\left(\frac{a_{1}\left(a_{1}+\mathscr{F}_{\rho, \sigma}^{\lambda}\left(a_{2}-a_{1}\right)\right)}{a_{1}+t_{1} \mathscr{F}_{\rho, \sigma}^{\lambda}\left(a_{2}-a_{1}\right)}\right)^{2 p \widehat{\alpha}}\left(d t_{1}\right)^{\widehat{\alpha}} \\
= & \left(a_{1}+\mathscr{F}_{\rho, \sigma}^{\lambda}\left(a_{2}-a_{1}\right)\right)^{2 p \widehat{\alpha}}\left[\frac{\Gamma(1+(p+2) \widehat{\alpha})}{\Gamma(1+(p+3) \widehat{\alpha})_{2}} \bar{F}_{1}^{\alpha}\right. \\
& \times\left(2 p, 1 ; p+2 ; \frac{-\mathscr{F}_{\rho, \sigma}^{\lambda}\left(a_{2}-a_{1}\right)}{a_{1}}\right)-\Gamma(1+\widehat{\alpha})_{2} \bar{F}_{1}^{\widehat{\alpha}} \\
& \times\left(2 p, 1 ; 2 ; \frac{-\mathscr{F}_{\rho, \sigma}^{\lambda}\left(a_{2}-a_{1}\right)}{a_{1}}\right)+(1-\omega)^{p \widehat{\alpha}} \frac{\Gamma(1+(p+1) \widehat{\alpha})}{\Gamma(1+(p+2) \widehat{\alpha})} \\
& \left.\times{ }_{2} \bar{F}_{1}^{\widehat{\alpha}}\left(2 p, p+1 ; p+2 ; \frac{-\mathscr{F}_{\rho, \sigma}^{\lambda}\left(a_{2}-a_{1}\right)}{a_{1}}\right)\right] .
\end{aligned}
$$

A combination of (55)-(58) with (54), we get the desired inequality (52). Hence, the proof is completed.

From Theorem 22, we get the following corollaries immediately.

Corollary 23. Let $\omega=0$. Then, Theorem 17 leads to the following local fractional integral inequality of "Ostrowski type" 


$$
\begin{aligned}
& \mid Q\left(a_{2}\right)-\left(\frac{\mathscr{F}_{\rho, \sigma}^{\lambda}\left(a_{2}-a_{1}\right)}{a_{1}\left(a_{1}+\mathscr{F}_{\rho, \sigma}^{\lambda}\left(a_{2}-a_{1}\right)\right)}\right)^{\widehat{\alpha}} \Gamma(1+\widehat{\alpha})_{a_{1}} \mathcal{F}_{a_{1}+\mathscr{F}_{\rho, \sigma}^{\lambda}\left(a_{2}-a_{1}\right)}^{(\widehat{\alpha})} \frac{Q(z)}{z^{2 \widehat{\alpha}} \mid} \\
& \leq \frac{\left(\mathscr{F}_{\rho, \sigma}^{\lambda}\left(a_{2}-a_{1}\right)\right)^{\widehat{\alpha}}}{a_{1}^{\widehat{\alpha}}\left(a_{1}+\mathscr{F}_{\rho, \sigma}^{\lambda}\left(a_{2}-a_{1}\right)\right)^{\widehat{\alpha}}}\left(\frac{\Gamma(1+s \widehat{\alpha})}{\Gamma(1+(s+1) \widehat{\alpha})}\right)^{1 / q} \\
& \quad \times\left\{\left(\Lambda_{5}^{\widehat{\alpha}}\left(p, 0, a_{1}, a_{2} ; s\right)\right)^{1 / p}\left[\left|Q^{(\widehat{\alpha})}\left(a_{1}\right)\right|^{q}+\left|Q^{(\widehat{\alpha})}\left(a_{2}\right)\right|^{q}\right]^{1 / q},\right.
\end{aligned}
$$

where

$$
\begin{aligned}
\Lambda_{5}^{\widehat{\alpha}}\left(p, 0, a_{1}, a_{2} ; s\right) \\
:=\left(a_{1}+\mathscr{F}_{\rho, \sigma}^{\lambda}\left(a_{2}-a_{1}\right)\right)^{2 p \widehat{\alpha}} \frac{\Gamma(1+r \widehat{\alpha})}{\Gamma(1+(r+1) \widehat{\alpha})_{2}} \bar{F}_{1}^{\widehat{\alpha}} \\
\quad\left(2 p, p+1 ; p+2 ; \frac{-\mathscr{F}_{\rho, \sigma}^{\lambda}\left(a_{2}-a_{1}\right)}{a_{1}}\right) .
\end{aligned}
$$

Corollary 24. Let $\omega=1$. Then, Theorem 17 leads to the following local fractional integral inequality of "Ostrowski type"

$$
\begin{aligned}
& \left|Q\left(a_{1}\right)-\left(\frac{\mathscr{F}_{\rho, \sigma}^{\lambda}\left(a_{2}-a_{1}\right)}{a_{1}\left(a_{1}+\mathscr{F}_{\rho, \sigma}^{\lambda}\left(a_{2}-a_{1}\right)\right)}\right)^{\widehat{\alpha}} \Gamma(1+\widehat{\alpha})_{a_{1}} \mathscr{F}_{a_{1}+\mathscr{F}_{\rho, \sigma}^{\lambda}\left(a_{2}-a_{1}\right)}^{(\widehat{\alpha})} \frac{Q(z)}{z^{2 \widehat{\alpha}}}\right| \\
& \leq \frac{\left(\mathscr{F}_{\rho, \sigma}^{\lambda}\left(a_{2}-a_{1}\right)\right)^{\widehat{\alpha}}}{a_{1}^{\widehat{\alpha}}\left(a_{1}+\mathscr{F}_{\rho, \sigma}^{\lambda}\left(a_{2}-a_{1}\right)\right)^{\widehat{\alpha}}}\left(\frac{\Gamma(1+s \widehat{\alpha})}{\Gamma(1+(s+1) \widehat{\alpha})}\right)^{1 / q} \\
& \quad \times\left\{( \Lambda _ { 6 } ^ { \widehat { \alpha } } ( p , 1 , a _ { 1 } , a _ { 2 } ; s ) ) ^ { 1 / p } \left[\left|Q^{(\widehat{\alpha})}\left(a_{1}\right)\right|^{q}\right.\right. \\
& \left.\left.\quad+\left|Q^{(\widehat{\alpha})}\left(a_{2}\right)\right|^{q}\right]^{1 / q}\right\},
\end{aligned}
$$

where

$$
\begin{aligned}
\Lambda_{6}^{\widehat{\alpha}}\left(p, 1, a_{1}, a_{2} ; s\right) \\
:=\left(a_{1}+\mathscr{F}_{\rho, \sigma}^{\lambda}\left(a_{2}-a_{1}\right)\right)^{2 \hat{p} \widehat{\alpha}}\left[\frac{\Gamma(1+(p+2) \widehat{\alpha})}{\Gamma(1+(p+3) \widehat{\alpha})_{2}} \bar{F}_{1}^{\widehat{\alpha}}\right. \\
\\
\cdot\left(2 p, 1 ; p+2 ; \frac{-\mathscr{F}_{\rho, \sigma}^{\lambda}\left(a_{2}-a_{1}\right)}{a_{1}}\right)-\Gamma(1+\widehat{\alpha})_{2} \bar{F}_{1}^{\widehat{\alpha}} \\
\left.\quad\left(2 p, 1 ; 2 ; \frac{-\mathscr{F}_{\rho, \sigma}^{\lambda}\left(a_{2}-a_{1}\right)}{a_{1}}\right)\right] .
\end{aligned}
$$

Corollary 25. By means of Corollary 23 and Corollary 24. Then, Theorem 22 leads to the following local fractional integral inequality of "trapezoid type"

$$
\begin{aligned}
& \mid \frac{Q\left(a_{1}\right)+Q\left(a_{2}\right)}{2^{\widehat{\alpha}}}-\left(\frac{\mathscr{F}_{\rho, \sigma}^{\lambda}\left(a_{2}-a_{1}\right)}{a_{1}\left(a_{1}+\mathscr{F}_{\rho, \sigma}^{\lambda}\left(a_{2}-a_{1}\right)\right)}\right)^{\widehat{\alpha}} \Gamma \\
& \quad \times(1+\widehat{\alpha})_{a_{1}} \mathscr{J}_{a_{1}+\mathscr{F}_{\rho, \sigma}^{\lambda}\left(a_{2}-a_{1}\right)}^{(\widehat{\alpha})} \frac{Q(z)}{z^{2 \alpha}} \mid \\
& \leq \frac{\left(\mathscr{F}_{\rho, \sigma}^{\lambda}\left(a_{2}-a_{1}\right)\right)^{\widehat{\alpha}}}{2^{\widehat{\alpha}} a_{1}^{\widehat{\alpha}}\left(a_{1}+\mathscr{F}_{\rho, \sigma}^{\lambda}\left(a_{2}-a_{1}\right)\right)^{\widehat{\alpha}}}\left(\frac{\Gamma(1+s \widehat{\alpha})}{\Gamma(1+(s+1) \widehat{\alpha})}\right)^{1 / q} \\
& \quad \times\left\{\left[\Lambda_{5}^{\widehat{\alpha}}\left(p, 0, a_{1}, a_{2} ; s\right)+\Lambda_{6}^{\widehat{\alpha}}\left(p, 1, a_{1}, a_{2} ; s\right)\right]\right. \\
& \left.\quad \times\left[\left|Q^{(\widehat{\alpha})}\left(a_{1}\right)\right|^{q}+\left|Q(\widehat{\alpha})\left(a_{2}\right)\right|^{q}\right]^{1 / q}\right\} .
\end{aligned}
$$

Corollary 26. Let $\omega=1 / 2$. Then, Theorem 17 leads to the following local fractional integral inequality of "midpoint type"

$$
\begin{aligned}
& \mid Q\left(\frac{2 a_{1} a_{2}}{a_{1}+a_{2}}\right)-\left(\frac{\mathscr{F}_{\rho, \sigma}^{\lambda}\left(a_{2}-a_{1}\right)}{a_{1}\left(a_{1}+\mathscr{F}_{\rho, \sigma}^{\lambda}\left(a_{2}-a_{1}\right)\right)}\right)^{\widehat{\alpha}} \Gamma \\
& \times(1+\widehat{\alpha})_{a_{1}} \mathcal{F}_{a_{1}+\mathcal{F}_{p, \sigma}^{\Re}\left(a_{2}-a_{1}\right)}^{(\widehat{Q})} \frac{Q(z)}{z^{2 \widehat{\alpha}}} \mid \\
& \leq \frac{\left(\mathscr{F}_{\rho, \sigma}^{\lambda}\left(a_{2}-a_{1}\right)\right)^{\widehat{\alpha}}}{a_{1}^{\widehat{\alpha}}\left(a_{1}+\mathscr{F}_{\rho, \sigma}^{\lambda}\left(a_{2}-a_{1}\right)\right)^{\widehat{\alpha}}}\left(\frac{\Gamma(1+s \widehat{\alpha})}{\Gamma(1+(s+1) \widehat{\alpha})}\right)^{1 / q} \\
& \times\left\{( \Lambda _ { 5 } ^ { \widehat { \alpha } } ( p , \frac { 1 } { 2 } , a _ { 1 } , a _ { 2 } ; s ) ) ^ { 1 / p } \left[\left(\frac{1}{2}\right)^{(s+1) \widehat{\alpha}}\left|Q^{(\widehat{\alpha})}\left(a_{1}\right)\right|^{q}\right.\right. \\
& \left.+\left(\frac{2^{(s+1) \widehat{\alpha}}-1^{\widehat{\alpha}}}{2^{(s+1) \widehat{\alpha}}}\right)\left|Q^{(\widehat{\alpha})}\left(a_{2}\right)\right|^{q}\right]^{1 / q}+\left(\Lambda_{6}^{\widehat{\alpha}}\left(p, \frac{1}{2}, a_{1}, a_{2} ; s\right)\right)^{1 / p} \\
& \left.\times\left[\left(\frac{2^{(s+1) \widehat{\alpha}}-1^{\widehat{\alpha}}}{2^{(s+1) \widehat{\alpha}}}\right)\left|Q^{(\widehat{\alpha})}\left(a_{1}\right)\right|^{q}+\left(\frac{1}{2}\right)^{(s+1) \widehat{\alpha}}\left|Q^{(\widehat{\alpha})}\left(a_{2}\right)\right|^{q}\right]^{1 / q}\right\},
\end{aligned}
$$

where

$$
\begin{aligned}
\Lambda_{5}^{\widehat{\alpha}}\left(p, \frac{1}{2}, a_{1}, a_{2} ; s\right) \\
:=\left(a_{1}+\mathscr{F}_{\rho, \sigma}^{\lambda}\left(a_{2}-a_{1}\right)\right)^{2 \widehat{\alpha \alpha}}\left(\frac{1}{2}\right)^{(p+1) \widehat{\alpha}} \frac{\Gamma(1+r \widehat{\alpha})}{\Gamma(1+(r+1) \widehat{\alpha})} \\
\quad \times{ }_{2} \bar{F}_{1}^{\widehat{\alpha}}\left(2 p, p+1 ; p+2 ; \frac{-\mathscr{F}_{\rho, \sigma}^{\lambda}\left(a_{2}-a_{1}\right)}{2 a_{1}}\right), \\
\widehat{\Lambda_{6}^{\widehat{\alpha}}}\left(p, \frac{1}{2}, a_{1}, a_{2} ; s\right) \\
:=\left(a_{1}+\mathscr{F}_{\rho, \sigma}^{\lambda}\left(a_{2}-a_{1}\right)\right)^{2 \hat{\alpha} \widehat{\alpha}}\left[\frac{\Gamma(1+(p+2) \widehat{\alpha})}{\Gamma(1+(p+3) \widehat{\alpha})_{2}} \bar{F}_{1}^{\widehat{\alpha}}\right.
\end{aligned}
$$




$$
\begin{aligned}
& \times\left(2 p, 1 ; p+2 ; \frac{-\mathscr{F}_{\rho, \sigma}^{\lambda}\left(a_{2}-a_{1}\right)}{a_{1}}\right)-\Gamma(1+\widehat{\alpha})_{2} \bar{F}_{1}^{\widehat{\alpha}} \\
& \times\left(2 p, 1 ; 2 ; \frac{-\mathscr{F}_{\rho, \sigma}^{\lambda}\left(a_{2}-a_{1}\right)}{a_{1}}\right)+\left(\frac{1}{2}\right)^{p \widehat{\alpha}} \frac{\Gamma(1+(p+1) \widehat{\alpha})}{\Gamma(1+(p+2) \widehat{\alpha})} \\
& \left.\times{ }_{2} \bar{F}_{1}^{\widehat{\alpha}}\left(2 p, p+1 ; p+2 ; \frac{-\mathscr{F}_{\rho, \sigma}^{\lambda}\left(a_{2}-a_{1}\right)}{a_{1}}\right)\right] .
\end{aligned}
$$

\section{Applications to Special Means}

In this section, we recall the following $\widehat{\alpha}$-type special means for two positive real numbers $a_{1}^{\widehat{\alpha}}$ and $a_{2}^{\widehat{\alpha}}$ where $a_{1}<a_{2}$ :

(1) The arithmetic mean

$$
\mathbb{A}_{\widehat{\alpha}}\left(a_{1}, a_{2}\right)=\frac{a_{1}^{\widehat{\alpha}}+a_{2}^{\widehat{\alpha}}}{2^{\widehat{\alpha}}}
$$

(2) The geometric mean

$$
\mathbb{G}_{\widehat{\alpha}}\left(a_{1}, a_{2}\right)=\sqrt{a_{1}^{\widehat{\alpha}} a_{2}^{\widehat{\alpha}}} .
$$

(3) The harmonic mean

$$
\mathrm{H}_{\widehat{\alpha}}\left(a_{1}, a_{2}\right)=\frac{\left(2 a_{1} a_{2}\right)^{\widehat{\alpha}}}{a_{1}^{\widehat{\alpha}}+a_{2}^{\widehat{\alpha}}}
$$

(4) The $r$-logarithmic mean

$\mathbb{L}_{r \widehat{\alpha}}\left(a_{1}, a_{2}\right)=\left(\frac{\Gamma(1+r \widehat{\alpha})}{\Gamma(1+(r+1) \widehat{\alpha})} \frac{a_{2}^{(r+1) \widehat{\alpha}}-a_{1}^{(r+1)} \widehat{\alpha}}{\left(a_{2}-a_{1}\right)^{\widehat{\alpha}}}\right)^{\frac{1}{r}}, r \in \mathbb{R} \backslash\{-1,0\}$.

These means have huge applications in pure and applied analysis and different type of numerical approximations. However, the following simple relationships are known in the literature.

$$
\mathrm{H}_{\widehat{\alpha}}\left(a_{1}, a_{2}\right) \leq \mathbb{G}_{\widehat{\alpha}}\left(a_{1}, a_{2}\right) \leq \mathbb{A}_{\widehat{\alpha}}\left(a_{1}, a_{2}\right) .
$$

Assume the mapping $W:(0, \infty) \mapsto \mathbb{R}^{\widehat{\alpha}}, W(z)=(\Gamma(1+$ $u \widehat{\alpha}) / \Gamma(1+(u+1) \widehat{\alpha})) z^{(s+1) \widehat{\alpha}}, z>0, s \geq 1$ and $r \geq 1$. Then, $\left|W^{(\widehat{\alpha})}(z)\right|^{r}=z^{s r \widehat{\alpha}}$ is generalized harmonically $\psi$-convex on $(0, \infty)$. Therefore, we can obtain the following results for $Q(z)=(\Gamma(1+u \widehat{\alpha}) / \Gamma(1+(u+1) \widehat{\alpha})) x^{(u+1) \widehat{\alpha}}$.
Proposition 27. For $0<a_{1}<a_{2}, u, r>1$ and $\omega \in[0,1]$ with $\widehat{\alpha} \in(0,1]$, we have the following inequality

$$
\begin{aligned}
\mid \mathbb{A}_{\widehat{\alpha}}\left(a_{1}^{u+1}, a_{2}^{u+1}\right)-\Gamma(1+\widehat{\alpha}) \mathbb{G}_{\widehat{\alpha}}^{2}\left(a_{1}, a_{1}+\mathscr{F}_{\rho, \sigma}^{\lambda}\left(a_{2}-a_{1}\right)\right) \mathbb{L}_{(u-1) \widehat{\alpha}}^{u-1} & \times\left(a_{1}, a_{1}+\mathscr{F}_{\rho, \sigma}^{\lambda}\left(a_{2}-a_{1}\right)\right) \mid \\
\leq & \frac{\Gamma(1+(u+1) \widehat{\alpha})}{\Gamma(1+u \widehat{\alpha})} \frac{\left(\mathscr{F}_{\rho, \sigma}^{\lambda}\left(a_{2}-a_{1}\right)\right)^{\widehat{\alpha}}}{\mathbb{G}_{\widehat{\alpha}}^{2}\left(2, a_{1},\left(a_{1}+\mathscr{F}_{\rho, \sigma}^{\lambda}\left(a_{2}-a_{1}\right)\right)\right)} \\
& \cdot\left(\frac{\Gamma(1+\widehat{\alpha})}{\Gamma(1+2 \widehat{\alpha})}\right)^{1-1 / q} \times\left[\left[\Lambda_{1}^{\widehat{\alpha}}\left(q, 0, a_{1}, a_{2} ; s\right) a_{1}^{q u \widehat{\alpha}}\right.\right. \\
& \left.+\Lambda_{2}^{\widehat{\alpha}}\left(q, 0, a_{1}, a_{2} ; s\right) a_{2}^{q u \widehat{\alpha}}\right]^{1 / q}+\left[\Lambda_{3}^{\alpha}\left(q, 1, a_{1}, a_{2} ; s\right) a_{1}^{q u \widehat{\alpha}}\right. \\
& \left.\left.+\Lambda_{4}^{\widehat{\alpha}}\left(q, 1, a_{1}, a_{2} ; s\right) a_{2}^{q u \hat{\alpha}}\right]^{1 / q}\right],
\end{aligned}
$$

where $\Lambda_{1}^{\widehat{\alpha}}\left(q, 0, a_{1}, a_{2} ; s\right), \Lambda_{2}^{\widehat{\alpha}}\left(q, 0, a_{1}, a_{2} ; s\right), \Lambda_{3}^{\widehat{\alpha}}\left(q, 1, a_{1}, a_{2}\right.$; $s)$, and $\Lambda_{4}^{\widehat{\alpha}}\left(q, 1, a_{1}, a_{2} ; s\right)$ are given in Corollary 18 and Corollary 19, respectively.

Proof. Taking $Q(z)=(\Gamma(1+u \widehat{\alpha}) / \Gamma(1+(u+1) \widehat{\alpha})) z^{(s+1) \widehat{\alpha}}, u$ $\geq 1$ for $z>0$ in Corollary 20, then we get the immediate consequence.

Proposition 28. For $0<a_{1}<a_{2}, s, q_{1}>1$ and $\omega \in[0,1]$ with $\widehat{\alpha} \in(0,1]$, we have the following inequality

$$
\begin{aligned}
\mid \mathbb{H}_{\widehat{\alpha}}^{s+1} & \left(a_{1}, a_{1}+\mathscr{F}_{\rho, \sigma}^{\lambda}\left(a_{2}-a_{1}\right)\right)-\Gamma(1+\widehat{\alpha}) \mathbb{G}_{\widehat{\alpha}}^{2} \\
& \times\left(a_{1}, a_{1}+\mathscr{F}_{\rho, \sigma}^{\lambda}\left(a_{2}-a_{1}\right)\right) \mathbb{L}_{(s-1) \widehat{\alpha}}^{s-1}\left(a_{1}, a_{1}+\mathscr{F}_{\rho, \sigma}^{\lambda}\left(a_{2}-a_{1}\right)\right) \mid \\
\leq & \frac{\Gamma(1+(u+1) \widehat{\alpha})}{\Gamma(1+u \widehat{\alpha})} \frac{\left(\mathscr{F}_{\rho, \sigma}^{\lambda}\left(a_{2}-a_{1}\right)\right)^{\widehat{\alpha}}}{\mathbb{G}_{\widehat{\alpha}}^{2}\left(a_{1}\left(a_{1}+\mathscr{F}_{\rho, \sigma}^{\lambda}\left(a_{2}-a_{1}\right)\right)\right)} \\
& \times\left(\frac{\Gamma(1+s \widehat{\alpha})}{\Gamma(1+(s+1) \widehat{\alpha})}\right)^{1 / q} \times\left\{\left(\Lambda_{5}^{\widehat{\alpha}}\left(p, \frac{1}{2}, a_{1}, a_{2} ; s\right)\right)^{1 / p}\right. \\
& \times\left[\left(\frac{1}{2}\right)^{(s+1) \widehat{\alpha}} a_{1}^{q u \hat{\alpha}}+\left(\frac{\left.\left.2^{(s+1) \widehat{\alpha}}-1^{1^{\alpha}}\right) a_{2}^{q u \hat{\alpha}}\right]^{1 / q}}{2^{(s+1) \widehat{\alpha}}}\right]^{1 / p}\left[\left(\frac{2^{(s+1) \widehat{\alpha}}-1^{\widehat{\alpha}}}{2^{(s+1) \widehat{\alpha}}}\right) a_{1}^{q u \widehat{\alpha}}\right.\right. \\
& +\left(\Lambda_{6}^{\widehat{\alpha}}\left(p, \frac{1}{2}, a_{1}, a_{2} ; s\right)\right) \\
& \left.\left.+\left(\frac{1}{2}\right)^{(s+1) \widehat{\alpha}} a_{2}^{q u \widehat{\alpha}}\right]^{1 / q}\right\},
\end{aligned}
$$

where $\Lambda_{5}^{\widehat{\alpha}}\left(p, 1 / 2, a_{1}, a_{2} ; s\right)$ and $\Lambda_{6}^{\widehat{\alpha}}\left(p, 1 / 2, a_{1}, a_{2} ; s\right)$ are defined in Corollary 26. 
Proof. Taking $Q(z)=(\Gamma(1+u \widehat{\alpha}) / \Gamma(1+(u+1) \widehat{\alpha})) z^{(u+1) \widehat{\alpha}}, u$ $\geq 1$ for $z>0$ in Corollary 26, then we get the immediate consequence.

\section{Applications to Mittag-Leffler Function}

Taking $\lambda=(1,1, \cdots$,$) with \rho=\delta_{1}, \mathfrak{R}\left(\delta_{1}\right)>0$ and $\sigma=1$, then from Theorems 13 to 22, the following results hold.

Theorem 29. Let $Q: \Omega_{\mathscr{F}}=\left[a_{1}, a_{1}+E_{\delta_{1}}\left(a_{2}-a_{1}\right)\right] \subset \mathbb{R} \backslash\{0\}$ $\mapsto \mathbb{R}^{\widehat{\alpha}}(0 \leq \widehat{\alpha}<1)$ be a generalized harmonically $\psi-s$-convex function where $a_{1}, a_{1}+E_{\delta_{1}}\left(a_{2}-a_{1}\right) \in \Omega_{\mathscr{F}}, E_{\delta_{1}}\left(a_{2}-a_{1}\right)>0$ such that $Q \in \mathscr{D}_{\widehat{\alpha}}\left[a_{1}, a_{1}+E_{\delta_{1}}\left(a_{2}-a_{1}\right)\right]$ and $Q^{(\widehat{\alpha})} \in \mathbb{C}_{\hat{\alpha}}\left[a_{1}, a_{1}\right.$ $\left.+E_{\delta_{1}}\left(a_{2}-a_{1}\right)\right]$. Then, the following inequalities hold:

$$
\begin{aligned}
& \frac{2^{(s-1) \widehat{\alpha}}}{\Gamma(1+\widehat{\alpha})} Q\left(\frac{2 a_{1}\left(a_{1}+E_{\delta_{1}}\left(a_{2}-a_{1}\right)\right)}{\left(2 a_{1}+E_{\delta_{1}}\left(a_{2}-a_{1}\right)\right)}\right) \\
& \quad \leq \frac{a_{1}^{\widehat{\alpha}}\left(a_{1}+E_{\delta_{1}}\left(a_{2}-a_{1}\right)\right)^{\widehat{\alpha}}}{\left(E_{\delta_{1}}\left(a_{2}-a_{1}\right)\right)^{\widehat{\alpha}}} \mathscr{J}_{a_{1}+E_{\delta_{1}}\left(a_{2}-a_{1}\right)}^{(\widehat{\alpha})} \frac{Q(z)}{z^{2 \widehat{\alpha}}} \\
& \quad \leq \frac{\Gamma(1+s \widehat{\alpha})}{\Gamma(1+(s+1) \widehat{\alpha})}\left[Q\left(a_{1}\right)+Q\left(a_{2}\right)\right] .
\end{aligned}
$$

Theorem 30. For $\rho, \sigma>0, \lambda=\{\lambda(m)\}_{m=0}^{\infty}$ a bounded sequence of real numbers and $Q: \mathscr{I}_{\mathscr{F}}^{\circ}=\left[a_{1}, a_{1}+E_{\delta_{1}}\left(a_{2}-a_{1}\right)\right] \subset \mathbb{R} \backslash\{0$ \}$\mapsto \mathbb{R}^{\widehat{\alpha}}(0<\widehat{\alpha} \leq 1)\left(\mathscr{F}_{\mathscr{F}}^{\circ}\right.$ is the interior of $\left.\mathscr{I}_{\mathscr{F}}\right)$, where $a_{1}, a_{1}$ $+E_{\delta_{1}}\left(a_{2}-a_{1}\right) \in \mathscr{F}_{\mathscr{F}}^{\circ}, E_{\delta_{1}}\left(a_{2}-a_{1}\right)>0$ such that $Q \in \mathscr{D}_{\widehat{\alpha}}\left[a_{1}\right.$, $\left.a_{1}+E_{\delta_{1}}\left(a_{2}-a_{1}\right)\right]$ and $Q^{(\widehat{\alpha})} \in \mathbb{C}_{\widehat{\alpha}}\left[a_{1}, a_{1}+E_{\delta_{1}}\left(a_{2}-a_{1}\right)\right]$. If $\left|Q^{(\widehat{\alpha})}\right|^{q_{1}}$ is a generalized harmonically $\psi-s$-convex function on $\mathscr{I}_{\mathscr{F}}$ for $p, q>1, p^{-1}+q^{-1}=1$. Then, the following inequality for local fractional integrals holds

$$
\begin{aligned}
& \left|\Omega_{Q}\left(a_{1}, a_{1}+E_{\delta_{1}}\left(a_{2}-a_{1}\right) ; \omega, \widehat{\alpha}\right)\right| \\
& \leq \frac{\left(E_{\delta_{1}}\left(a_{2}-a_{1}\right)\right)^{\widehat{\alpha}}}{a_{1}^{\widehat{\alpha}}\left(a_{1}+E_{\delta_{1}}\left(a_{2}-a_{1}\right)\right)^{\widehat{\alpha}}}\left(\frac{\Gamma(1+\widehat{\alpha})}{\Gamma(1+2 \widehat{\alpha})}\right)^{1-1 / q} \\
& \quad \times\left[\left\{( 1 - \omega ) ^ { 2 ( 1 - \frac { 1 } { q } ) \widehat { \alpha } } \left[\Lambda_{1}^{\widehat{\alpha}}\left(q, \omega, a_{1}, a_{2} ; s\right)\left|Q^{(\widehat{\alpha})}\left(a_{1}\right)\right|^{q}\right.\right.\right. \\
& \left.\left.\quad+\left.\Lambda_{2}^{\widehat{\alpha}}\left(q, \omega, a_{1}, a_{2} ; s\right)\left|Q^{(\widehat{\alpha})}\left(a_{2}\right)\right|\right|^{q}\right]\right\}^{1 / q} \\
& \quad+\left\{( \omega ) ^ { 2 ( 1 - \frac { 1 } { q } ) \widehat { \alpha } } \left[\Lambda_{3}^{\widehat{\alpha}}\left(q, \omega, a_{1}, a_{2} ; s\right)\left|Q^{(\widehat{\alpha})}\left(a_{1}\right)\right|^{q}\right.\right. \\
& \left.\left.\left.\quad+\Lambda_{4}^{\widehat{\alpha}}\left(q, \omega, a_{1}, a_{2} ; s\right)\left|Q^{(\widehat{\alpha})}\left(a_{2}\right)\right|^{q}\right]\right\}^{1 / q}\right],
\end{aligned}
$$

where

$$
\begin{aligned}
& \Lambda_{1}^{\widehat{\alpha}}\left(q, \omega, a_{1}, a_{2} ; s\right) \\
& :=(1-\omega)^{(s+2) \widehat{\alpha}}\left(a_{1}+E_{\delta_{1}}\left(a_{2}-a_{1}\right)\right)^{2 q \widehat{\alpha}} \\
& \times \frac{\Gamma(1+(s+1) \widehat{\alpha})}{\Gamma(1+(s+2) \widehat{\alpha})_{2}} \bar{F}_{1}^{\widehat{\alpha}}(2 q, s+2 ; s \\
& \left.+3 ; \frac{(\omega-1) E_{\delta_{1}}\left(a_{2}-a_{1}\right)}{a_{1}}\right), \\
& :=(1-\omega)^{2 \widehat{\alpha}}\left(a_{1}+E_{\delta_{1}}\left(a_{2}-a_{1}\right)\right)^{2 q \widehat{\alpha}} \frac{\Gamma(1+\widehat{\alpha})}{\Gamma(1+2 \widehat{\alpha})} \\
& \times{ }_{2} \bar{F}_{1}^{\widehat{\alpha}}\left(2 q, 2 ; 3 ; \frac{(\omega-1) E_{\delta_{1}}\left(a_{2}-a_{1}\right)}{a_{1}}\right) \\
& -\Lambda_{1}^{\widehat{\alpha}}\left(q, \omega, a_{1}, a_{2} ; s\right), \\
& :=\left(a_{1}+E_{\delta_{1}}\left(a_{2}-a_{1}\right)\right)^{2 q \widehat{\alpha}}\left[\frac{\Gamma(1+s \widehat{\alpha})}{\Gamma(1+(s+1) \widehat{\alpha})_{2}} \bar{F}_{1}^{\widehat{\alpha}}\right. \\
& \cdot\left(2 q, s+1 ; s+2 ; \frac{(\omega-1) E_{\delta_{1}}\left(a_{2}-a_{1}\right)}{a_{1}}\right) \\
& -\frac{\Gamma(1+(s+2) \widehat{\alpha})}{\Gamma(1+(s+3) \widehat{\alpha})_{2}} \widehat{F}_{1}^{\widehat{\alpha}}(2 q, s+2 ; s \\
& \left.+3 ; \frac{(\omega-1) E_{\delta_{1}}\left(a_{2}-a_{1}\right)}{a_{1}}\right)-(1-\omega)^{(s+1) \widehat{\alpha}} \\
& \frac{\Gamma(1+s \widehat{\alpha})}{\Gamma(1+(s+1) \widehat{\alpha})} \times{ }_{2} \bar{F}_{1}^{\widehat{\alpha}}(2 q,(s+1) ; \\
& \left.\left.(s+2) ; \frac{(\omega-1) E_{\delta_{1}}\left(a_{2}-a_{1}\right)}{a_{1}}\right)\right]+\Lambda_{1}^{\widehat{\alpha}}\left(q, \omega, a_{1}, a_{2} ; s\right),
\end{aligned}
$$

$\Lambda_{4}^{\widehat{\alpha}}\left(q, \omega, a_{1}, a_{2} ; s\right)$

$$
\begin{aligned}
:= & \left(a_{1}+E_{\delta_{1}}\left(a_{2}-a_{1}\right)\right)^{2 q \widehat{\alpha}}\left[\frac{\Gamma(1+(s+2) \widehat{\alpha})}{\Gamma(1+(s+3) \widehat{\alpha})_{2}} \bar{F}_{1}^{\widehat{\alpha}}\right. \\
& \cdot\left(2 q, 1 ; s+3 ; \frac{(\omega-1) E_{\delta_{1}}\left(a_{2}-a_{1}\right)}{a_{1}}\right) \\
& -(1-\omega)^{\widehat{\alpha}} \Gamma(1+\widehat{\alpha})_{2} \widehat{F}_{1}^{\widehat{\alpha}}(2 q, 1 ; 2 ; \\
& \left.\cdot \frac{(\omega-1) E_{\delta_{1}}\left(a_{2}-a_{1}\right)}{a_{1}}\right)+(1-\omega)^{(s+2) \widehat{\alpha}} \\
& \cdot \frac{\Gamma(1+(s+2) \widehat{\alpha})}{\Gamma(1+(s+3) \widehat{\alpha})_{2}} \bar{F}_{1}^{\widehat{\alpha}}(2 q, s+2 ; s \\
& \left.\left.+3 ; \frac{(\omega-1) E_{\delta_{1}}\left(a_{2}-a_{1}\right)}{a_{1}}\right)\right] .
\end{aligned}
$$

\section{Conclusion}

On the development of this work, we have introduced the notion of generalized harmonically $\psi$-convex functions in 
fractal domain. Adopting this novel approach, we derived a new identity that has been correlated with some novel and well-known midpoint type, Ostrowski type, and trapezoid type inequalities in the relative literature have been introduced. For generalized harmonically $\psi$-s-convex functions, we investigated bounds for Hermite-Hadamard type inequalities for local fractional differentiation in the frame of Raina's function, which direct to several noted estimates computed by [40]. In addition to this, the findings and the techniques in this work are entirely new. This study demonstrates methods that can be utilized to examine the much to explore in the area of local fractional calculus, geometric aided design by proposing the variation in the fractal parameters. Hence, the proposed methodology is a powerful tool for solving local fractional linear equations of physical importance.

\section{Data Availability}

The data used to support the findings of this study are available from the corresponding author uponThe data used to support the findings of this study are available from the corresponding author upon request.

\section{Conflicts of Interest}

The authors declare that they have no competing interests.

\section{Authors' Contributions}

All authors contributed equally to the writing of this paper. All authors read and approved the final manuscript.

\section{Acknowledgments}

This work was supported by the National Natural Science Foundation of China (Grant No. 61673169).

\section{References}

[1] S. Boccaletti, W. Ditto, G. Mindlin, and A. Atangana, "Modeling and forecasting of epidemic spreading: the case of Covid19 and beyond," Chaos, Solitons and Fractals, vol. 135, article 109794, 2020.

[2] S. Kumar and A. Atangana, "A numerical study of the nonlinear fractional mathematical model of tumor cells in presence of chemotherapeutic treatment," International Journal of Biomathematics, vol. 13, no. 3, article 2050021, 2020.

[3] B. Ghanbari and A. Atangana, "A new application of fractional Atangana-Baleanu derivatives: designing ABC- fractional masks in image processing," Physica A, vol. 542, article 123516, 2020.

[4] J. Danane, K. Allali, and Z. Hammouch, "Mathematical analysis of a fractional differential model of HBV infection with antibody immune response," Chaos, Solitons and Fractals, vol. 136, article 109787, 2020.

[5] W. Gao, P. Veeresha, D. G. Prakasha, and H. M. Baskonus, "Novel dynamic structures of 2019-nCoV with nonlocal operator via powerful computational technique," Biology, vol. 9, no. 5, p. 107, 2020.

[6] X. J. Yang, Advanced Local Fractional Calculus and Its Applications, World Science, New York, NY, USA, 2012.
[7] A. M. Yang, Y. Z. Zhang, and Y. Long, "The Yang-Fourier transforms to heat-conduction in a semi-infinite fractal bar," Thermal Science, vol. 17, no. 3, pp. 707-713, 2013.

[8] J. Singh, D. Kumar, and J. J. Nieto, "A reliable algorithm for a local fractional tricomi equation arising in fractal transonic flow," Entropy, vol. 18, no. 6, article 206, 2016.

[9] D. Kumar, F. Tchier, J. Singh, and D. Baleanu, "An efficient computational technique for fractal vehicular traffic flow," Entropy, vol. 20, no. 4, article 259, 2018.

[10] H. X. Mo and X. Sui, "Hermite-Hadamard-type inequalities for generalized s-convex functions on real linear fractal set $\mathbb{R}^{\alpha}(0<\alpha<1)$," The Mathematical Scientist, vol. 11, no. 3, pp. 241-246, 2017.

[11] T. Abdeljawad, S. Rashid, Z. Hammouch, and Y.-M. Chu, "Some new local fractional inequalities associated with generalized (s,m)-convex functions and applications," Advances in Difference Equations, vol. 2020, no. 1, Article ID 406, p. 27, 2020.

[12] W. Sun and Q. Liu, "Hadamard type local fractional integral inequalities for generalized harmonically convex functions and applications," Mathematical Methods in the Applied Sciences, vol. 43, no. 9, pp. 5776-5787, 2020.

[13] W. Sun, "Generalized harmonically convex functions on fractal sets and related Hermite-Hadamard type inequalities," The Journal of Nonlinear Sciences and Applications, vol. 10, no. 11, pp. 5869-5880, 2017.

[14] T. Abdeljawad, S. Rashid, Z. Hammouch, İ. İşcan, and Y. M. $\mathrm{Chu}$, "Some new Simpson-type inequalities for generalized pconvex function on fractal sets with applications," Advances in Difference Equations, vol. 2020, Article ID 496, 26 pages, 2020.

[15] G. Chen, H. M. Srivastava, P. Wang, and W. Wei, "Some further generalizations of Hölder's inequality and related results on fractal space," Abstract and Applied Analysis, vol. 2014, Article ID 832802, 7 pages, 2014.

[16] W. Wang and J. Qi, "Some new estimates of HermiteHadamard type inequalities for harmonically convex functions with applications," International Journal of Analysis and Applications, vol. 13, no. 1, pp. 15-21, 2017.

[17] M. E. Özdemir, Ç. Yildiz, and A. O. Akdemir, "On some inequalities for s-convex functions and applications," Journal of Inequalities and Applications, vol. 2013, no. 1, Article ID 333, 2013.

[18] S. Rashid, D. Baleanu, and Y.-M. Chu, "Some new extensions for fractional integral operator having exponential in the kernel and their applications in physical systems," Open Physics, vol. 18, no. 1, pp. 478-491, 2020.

[19] S. Rashid, T. Abdeljawad, F. Jarad, and M. A. Noor, "Some estimates for generalized Riemann-Liouville fractional integrals of exponentially convex functions and their applications," Mathematics, vol. 7, no. 9, p. 807, 2019.

[20] S. Rashid, H. Kalsoom, Z. Hammouch, R. Ashraf, D. Baleanu, and Y.-M. Chu, "New multi-parametrized estimates having pth-order differentiability in fractional calculus for predominating $\hbar$-convex functions in Hilbert space," Symmetry, vol. 12, no. 2, p. 222, 2020.

[21] S. Varosanec, "On_h_-convexity," Journal of Mathematical Analysis and Applications, vol. 326, no. 1, pp. 303-311, 2007.

[22] W. W. Breckner, "Stetigkeitsaussagen fur eine Klasse verallgemeinerter konvexer funktionen in topologischen linearen 
Raumen," Publications de l'Institut Mathématique, vol. 23, pp. 13-20, 1978.

[23] P. O. Mohammed and T. Abdeljawad, "Integral inequalities for a fractional operator of a function with respect to another function with nonsingular kernel," Advances in Difference Equations, vol. 2020, no. 1, 2020.

[24] Y. Li, S. Rashid, Z. Hammouch, D. Baleanu, and Y.-M. Chu, "New Newton's type estimates pertaining to local fractional integral via generalized p-convexity with applications," Fractals, p. 2140016, 2021.

[25] M. Al Qurashi, S. Rashid, A. Khalid, Y. Karaca, and Y.-M. Chu, "New computations of Ostrowski type inequality pertaining to fractal style with applications," Fractals, 2021.

[26] Z. Khan, S. Rashid, R. Ashraf, D. Baleanu, and Y.-M. Chu, "Generalized trapezium-type inequalities in the settings of fractal sets for functions having generalized convexity property," Advances in Difference Equations, vol. 2020, 24 pages, 2020.

[27] P. O. Mohammed, T. Abdeljawad, and A. Kashuri, "Fractional Hermite-Hadamard-Fejer inequalities for a convex function with respect to an increasing function involving a positive weighted symmetric function," Symmetry, vol. 12, no. 9, p. 1503, 2020.

[28] Y. M. Chu, M. A. Khan, T. Ali, and S. S. Dragomir, "Inequalities for $\alpha^{\wedge}$-fractional differentiable functions," Journal of Inequalities and Applications, vol. 2017, 12 pages, 2017.

[29] S. Rashid, İ. İşcan, D. Baleanu, and Y. M. Chu, "Generation of new fractional inequalities via n-polynomials s-type convexity with applications," Advances in Difference Equations, vol. 2020, 20 pages, 2020.

[30] C. P. Niculescu and L. E. Persson, Convex Functions and Their Applications, Springer, New York, 2006.

[31] C. Hermite, "Sur deux limites d'une intégrale définie," Mathesis, vol. 3, no. 82, 1883 .

[32] J. Hadamard, "Etude sur les proprietes des fonctions entieres et en particulier d'une fonction considree par Riemann," Journal de mathématiques pures et appliquées, vol. 58, pp. 171-215, 1893.

[33] M. J. Vivas-Cortez, R. Liko, A. Kashuri, and J. E. Hernández Hernández, "New quantum estimates of trapezium-type inequalities for generalized $\phi$-convex functions," Mathematics, vol. 7, no. 11, p. 1047, 2019.

[34] Y. Khurshid, M. Adil Khan, and Y.-M. Chu, "Conformable fractional integral inequalities for $<\mathrm{i}>\mathrm{GG}</ \mathrm{i}>$ - and $<$ i $>$ GA $</$ i $>$-convex functions," AIMS Math, vol. 5, no. 5, pp. 5012-5030, 2020.

[35] I. I. Scan, "Hermite-Hadamard type inequalities for harmonically convex functions," Hacettepe Journal of Mathematics and statistics, vol. 43, no. 6, pp. 935-942, 2014.

[36] F. Qi, P. O. Mohammed, J. C. Yao, and Y. H. Yao, “Generalized fractional integral inequalities of Hermite-Hadamard type for $\left(\alpha^{\wedge}, \mathrm{m}\right)$-convex functions," Journal of Inequalities and Applications, vol. 2019, 7 pages, 2019.

[37] A. Atangana, "Modelling the spread of COVID-19 with new fractal-fractional operators: can the lockdown save mankind before vaccination?," Chaos, Solitons and Fractals, vol. 136, article 109860, 2020.

[38] R. K. Raina, “On generalized Wright's hypergeometric functions and fractional calculus operators," East Asian mathematical journal, vol. 21, no. 2, pp. 191-203, 2005.
[39] I. I. Scan, "Ostrowski type inequalities for harmonically sconvex functions," 2013, https://arxiv.org/abs/1307.5201.

[40] S. S. Zhou, S. Rashid, S. Parveen, A. O. Akdemir, and Z. Hammouch, "New computations for extended weighted functionals within the Hilfer generalized proportional fractional integral operators," AIMS Mathematics, vol. 6, no. 5, pp. 4507-4525, 2020. 